\title{
Photoluminescence of hydrogenated amorphous carbons
}

\section{Wavelength-dependent yield and implications for the extended red emission ${ }^{\star}$}

\author{
M. Godard ${ }^{\star \star}$ and E. Dartois
}

\author{
Institut d'Astrophysique Spatiale (IAS), UMR8617, Université Paris-Sud, bâtiment 121, 91405 Orsay Cedex, France \\ e-mail: marie.godard@ias.u-psud.fr
}

Received 18 December 2009 / Accepted 11 March 2010

\begin{abstract}
Context. Hydrogenated amorphous carbons (a-C:H or HAC) have proved to be excellent analogs of interstellar dust observed in galaxies diffuse interstellar medium (DISM) through infrared vibrational absorption bands $(3.4 \mu \mathrm{m}, 6.8 \mu \mathrm{m}$, and $7.2 \mu \mathrm{m}$ bands). They exhibit photoluminescence (PL) after excitation by UV-visible photons, and are possible carriers for the extended red emission (ERE), a broad red emission band observed in various interstellar environments.

Aims. As many candidate materials/molecules can photoluminesce in the visible, along with the carrier abundance, the PL efficiency represents one of the strongest constraints set by such ERE observations. We wish to precisely characterize the PL behavior of a-C:H as a family of materials.

Methods. The a-C:H samples are produced in the form of films deposited on substrates by plasma-enhanced chemical vapor deposition. The produced films were analyzed in transmission by UV-visible and IR spectroscopy, and the wavelength dependent PL spectra were recorded. The intrinsic absolute quantum yield $\eta$ was then rigorously calculated taking self-absorption of the PL by the film and interfaces effects into account.

Results. A wide range of different laboratory synthesized a-C:H were analyzed. Their PL properties are dependent on the optical gap $E_{04}$ : when $E_{04}$ decreases from $4.3 \mathrm{eV}$ to $2.8 \mathrm{eV}$, the a-C:H vary from highly ( $\eta \sim 1 \%$ ) yellow photoluminescent soft materials to hard materials that emit a wider PL band in the red spectral range, with a lower efficiency $(\eta \sim 0.01-0.1 \%)$. For any given a-C:H, the PL characteristics (central wavelength, band width and efficiency) are found to be essentially constant over the explored excitation range $\left(\lambda_{\text {exc }} \gtrsim 250 \mathrm{~nm}\right)$. We compared the characteristics of the produced interstellar dust analog to the constraints imposed by the ERE observations.

Conclusions. As for ERE observations, PL efficiencies and band widths of a-C:H are both correlated to the PL central wavelengths. The excitation responsible for the a-C:H emission is efficient over a wide spectral range that matches the ERE excitation. The present a-C:H encounter difficulties for the diffuse ISM ERE observations $(\eta \geq 10 \%)$ in simultaneously satisfying the high quantum yield criteria and PL spectral characteristics. We still need to investigate the role of a small number of residual oxygen atoms in the laboratory-produced a-C:H network in quenching the PL yield, as well as to consider the interstellar temperature effect for our analogs.
\end{abstract}

Key words. dust, extinction - radiation mechanisms: non-thermal - infrared: ISM - ISM: lines and bands - astrochemistry methods: laboratory

\section{Introduction}

The extended red emission (ERE), first observed in the Red Rectangle nebula (Cohen et al. 1975; Schmidt et al. 1980), is a large (quartile flux width $\Delta q^{1}$ between 60 and $120 \mathrm{~nm}$ ) featureless emission band in the red part of the visible spectrum (between 540 and $950 \mathrm{~nm}$ ) observed in a wide range of astrophysical environments: reflection nebulae (Witt \& Schild 1985; Witt \& Boroson 1990), carbon-rich planetary nebulae (Furton \& Witt 1990, 1992), a dark nebula (Mattila 1979; Chlewicki \& Laureijs

* Appendices are only available in electronic form at http: //www . aanda.org

$\star \star$ Part of the equipment used in this work has been financed by the French INSU-CNRS program, the Physique et Chimie du Milieu Interstellaire (PCMI). This research has made use of NASA's Astrophysics Data System.

1 This quartile flux width $\Delta q$ is the difference between the third and first quartiles of the band flux (as defined by Witt \& Boroson 1990). Note that $\Delta q$ is much lower than the full width at half maximum $(F W H M)$. In the case of a Gaussian shape: $\Delta q=0.57 \cdot F W H M$.
1987), H II regions (Perrin \& Sivan 1992; Sivan \& Perrin 1993; Darbon et al. 1998, 2000), diffuse interstellar medium (DISM) (Szomoru \& Guhathakurta 1998; Gordon et al. 1998; Witt et al. 2008), and in external galaxies (Perrin et al. 1995; Darbon et al. 1998; Pierini et al. 2002). ERE is thus a general phenomenon.

This spectral feature is commonly attributed to the photoluminescence (PL) of interstellar dust following the absorption of UV-visible photons, but the true nature of the ERE carriers is still under debate. Many candidates have been proposed over the past decades: polycyclic aromatic hydrocarbon (PAH) molecules, ions or clusters (D'Hendecourt et al. 1986; Vijh et al. 2005; Rhee et al. 2007; Berné et al. 2008), hydrogenated amorphous carbons (HAC or a-C:H) (Duley 1985; Duley \& Williams 1988; Witt \& Schild 1988; Witt \& Boroson 1990; Furton \& Witt 1993; Duley et al. 1997; Seahra \& Duley 1999), quenched carbonaceous composites (QCC) (Sakata et al. 1992; Wada et al. 2009), nanodiamonds (Duley 1988; Chang et al. 2006), $\mathrm{C}_{60}$ (Webster 1993), and also non carbon-based materials such as crystalline silicon nanoparticules (SNP) (Ledoux et al. 1998; Witt et al. 1998; Ledoux et al. 2001; Smith \& Witt 2002). 
Recently, Duley (2009) have suggested that ERE may not be exclusively caused by PL but may result from a combination of PL and carbon clusters/dehydrogenated carbon molecules thermal emission. Up to now, none of these candidates have been clearly identified as the ERE carrier material.

To identify which interstellar material is responsible for this observed large emission band, or, at least, narrow the range of candidates, each constraint imposed by the ERE observations must be carefully compared to the properties of ERE carrier candidates. An example of an observational relationship that should help identify the ERE carriers is the correlation found between the width and peak wavelength of ERE (Witt \& Boroson 1990; Darbon et al. 1999): the band width $\Delta q$ increases from 60 to $120 \mathrm{~nm}$ as the ERE maximum position varies from 650 to $780 \mathrm{~nm}$. In addition to the ERE positions and shapes, the observational constraints, reviewed by Smith \& Witt (2002) and Witt \& Vijh (2004), set a lower limit on the PL quantum yield of the ERE. This quantum yield $\eta$ is defined as the ratio of the emitted and the absorbed (by the ERE carriers) photon numbers. This yield is the quantum yield and must not be confused with the yield defined as an energy ratio. The quantum yield can be greater than one in the case of more than one ERE photon emission after an UV-visible photon absorption. It is not straightforward to evaluate the ERE quantum yield from observations (Smith \& Witt 2002). Some hypothesis have to be assumed about the excitation range that causes the ERE and the way the amount of absorbed light is determined from dustscattered light (i.e. assuming the dust albedo and extinction law wavelength dependence, the illuminating star's spectral energy distribution and the geometry of the nebula as well known parameters). The determined absorption accounts for all kinds of intervening molecules and solids, not only ERE carriers. Thus, the observed quantum yields set a lower limit on the ERE efficiency. The efficiency lower limits reviewed by Smith \& Witt (2002) lie between about $0.1 \%$ and up to $10 \%$ depending on the interstellar environments (quantum efficiency as high as $20 \%$ have been estimated by the same method for ERE in the Red Rectangle nebula, which is treated apart). They find that ERE efficiency tends to decline with the increase of local radiation field intensity.

Amorphous hydrogenated carbons (a-C:H or HAC) are one of the ERE carrier candidates. They are carbonaceous material consisting of a mixture of $\mathrm{sp}^{2}$ and $\mathrm{sp}^{3}$ hybridized bonds. The $\mathrm{a}-\mathrm{C}: \mathrm{H}$ are of astrophysical interest since they have proved to be analogs of one of the interstellar dust components (e.g. Sandford et al. 1991; Pendleton \& Allamandola 2002; Spoon et al. 2004; Dartois et al. 2005) through IR absorption bands (C-H stretching modes at $3.4 \mu \mathrm{m}$ and $\mathrm{C}-\mathrm{H}$ bending modes at 6.85 and $7.25 \mu \mathrm{m})$ ubiquitously observed in the diffuse interstellar medium of both our Galaxy and of other galaxies. This material represents a highly significant dust component of galaxies, since 5 to $30 \%$ of the total available cosmic carbon (Sandford et al. 1991; Duley et al. 1998; Dartois et al. 2005) is contained in the $3.4 \mu \mathrm{m}$ feature carrier.

Amorphous hydrogenated carbons photoluminesce in the visible spectral range. Characterization of the photoluminescence behavior of this interstellar component is thus required to evaluate its contribution to the interstellar dust emission, and it is especially important to compare the a-C:H PL to the ERE observed properties. In particular, the PL efficiency, which is one of the strongest constraints set by the observations for the ERE carrier candidates as described above, has to be accurately investigated for the a-C:H materials. Until now, most of the works about the photoluminescence efficiencies of astrophysical relevant a-C:H provided results in terms of relative efficiencies (e.g. Rusli et al. 1996) and only for a few excitation wavelengths. The few results for absolute a-C:H PL efficiencies (Furton \& Witt 1993; Furton et al. 1999; Ledoux et al. 2001) vary over four orders of magnitude. In addition, optical effects modifying the apparent efficiency as compared to the intrinsic efficiency are not properly taken into account.

Carefully studying the PL absolute efficiency of amorphous hydrogenated carbons is the aim of this work. In this article, we present the first results of a systematic a-C:H photoluminescence study, which has the advantage of being a multi-wavelength excitation study that properly takes optical effects in the emitting thin film into account. We describe in Sect. 2 the experimental setups allowing the production and analysis of the a-C:H samples. Section 3 describes how the a-C:H PL absolute quantum yield is calculated from the PL measurements (detailed calculations in appendixes) and the obtained results (concerning PL yields and spectra) are put in perspective with previous relative efficiencies measurements in Sect. 4. These results and their astrophysical implications about ERE are then discussed in Sect. 5, followed by our conclusion in Sect. 6 .

\section{Experiments}

\subsection{Film preparation}

The interstellar a-C:H analogs were produced using a plasmaenhanced chemical vapor deposition (PECVD) system: a plasma of a hydrocarbon precursor gas is created in a vacuum chamber. The pressure during deposition was maintained in the range between $10^{-3}$ and $10^{-1}$ mbar. As the precursor gas, we used different hydrocarbons (methane, acetylene, butadiene, phenylacetylene, isobutane, toluene, and limonene) that can also be mixed with molecular hydrogen or argon. The addition of an inert gas diluent such as argon allows us to stabilize the plasma or to change deposition conditions (Seth \& Babu 1993). The C/H ratio and the hybridization type vary from one hydrocarbon precursor to another one and have some influence on the produced a-C:H (Schwarz-Selinger et al. 1999).

The plasma is created via an Evenson cavity used to excite microwave discharges in molecular precursors with a $2450 \mathrm{MHz}$ radio-frequency microwave generator. The film is formed by deposition of the radicals and ions resulting from the precursor on a substrate (quartz or $\mathrm{KBr}$, for example) located in the vacuum chamber. A negative electric potential can be applied to a grid placed just in front of the substrate in order to accelerate the ions coming from the plasma. By applying different voltages, thus varying the energy of the impinging ions, we can explore a wider range of produced a-C:H films (Schwarz-Selinger et al. 1999; Rusli et al. 1996). The deposition time to obtain a few micrometers thick film is variable, from a few minutes to a few hours depending on the deposition conditions. The a-C:H film obtained can then be analyzed ex-situ.

\subsection{Film characterization: structure and optical constants}

\subsubsection{Infrared spectroscopy}

The IR ex situ analysis of the samples were performed either by FTIR spectroscopy with a Bruker IFS66V infrared spectrometer or by FTIR micro-spectroscopy using a Nicolet Magna-IR 560 ESP spectrometer coupled to a Nicolet Nicplan infrared microscope located at the synchrotron SOLEIL (the microscope enables us to check the sample planarity). The transmission spectra 
M. Godard and E. Dartois: Photoluminescence of hydrogenated amorphous carbons
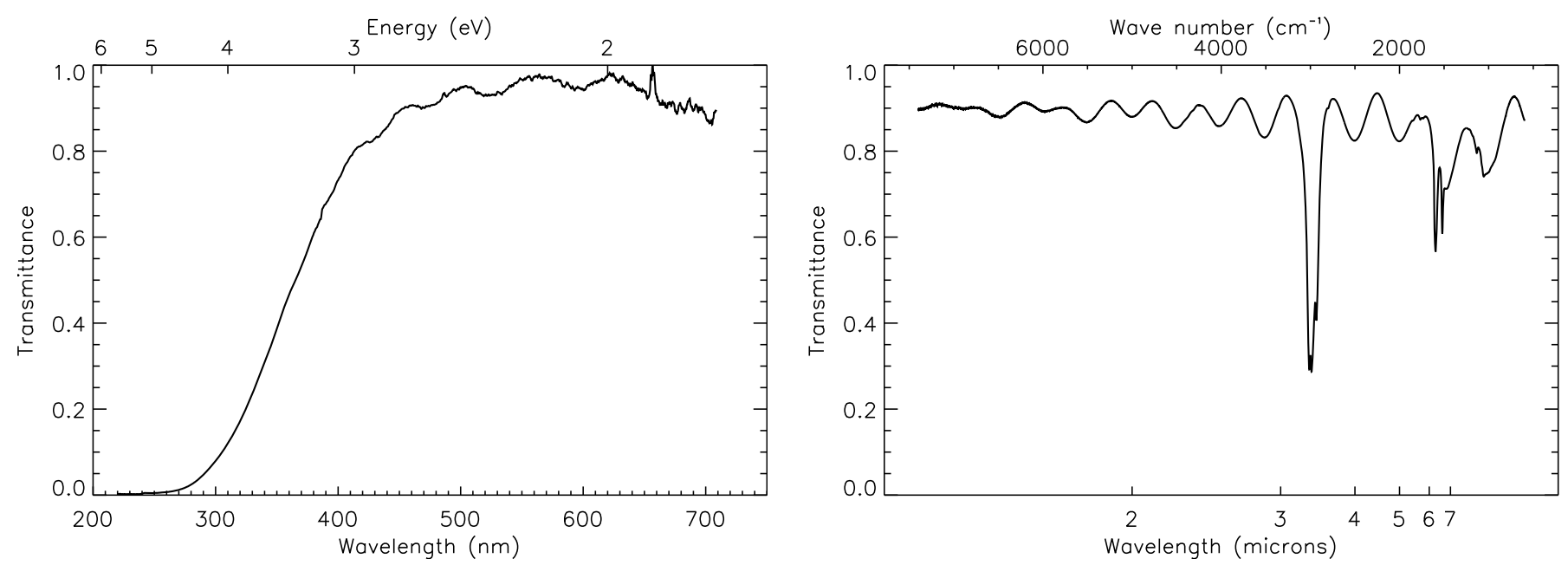

Fig. 1. Examples of transmission spectra in the UV-visible (left) and IR region (right) of a typically produced a-C:H film.

Table 1. a-C:H vibrational assignments.

\begin{tabular}{ccl}
\hline \hline $\begin{array}{c}\text { Position } \sigma \\
\left(\mathrm{cm}^{-1}\right)\end{array}$ & $\begin{array}{c}\text { Position } \lambda \\
(\mu \mathrm{m})\end{array}$ & Vibrational Mode \\
\hline 3300 & 3.03 & alkyne $\mathrm{CH}$ stretching \\
3078 & 3.25 & olefinic $\mathrm{CH}_{2}$ asymmetric stretching \\
3050 & 3.28 & aromatic $\mathrm{CH}$ stretching \\
3010 & 3.32 & olefinic $\mathrm{CH}$ stretching \\
2955 & 3.38 & aliphatic $\mathrm{CH}_{3}$ asymmetric stretching \\
2925 & 3.42 & aliphatic $\mathrm{CH}_{2}$ asymmetric stretching \\
2870 & 3.48 & aliphatic $\mathrm{CH}_{3}$ symmetric stretching \\
2855 & 3.50 & aliphatic $\mathrm{CH}_{2}$ symmetric stretching \\
2100 & 4.76 & alkyne $\mathrm{CC}$ stretching \\
1900 & 5.26 & $\mathrm{C}=\mathrm{C}=\mathrm{C}_{\text {stretching }}$ \\
$1550-1650$ & $6.0-6.4$ & $\mathrm{C}=\mathrm{C}$ stretching \\
1450 & 6.89 & aliphatic $\mathrm{CH}_{2}$ bending \\
1375 & 7.27 & aliphatic $\mathrm{CH}_{3}$ symmetric bending \\
970 & 10.3 & $\mathrm{CH}=\mathrm{CH}_{\text {out }}$ of plane bending \\
$890-910$ & $10.9-11.2$ & $\mathrm{CH}=\mathrm{CH}_{2}$ out of plane bending \\
\hline
\end{tabular}

were recorded in the $7500-400 \mathrm{~cm}^{-1}$ range (when using $\mathrm{KBr}$ as substrate) with a resolution of $2 \mathrm{~cm}^{-1}$. An example of produced $\mathrm{a}-\mathrm{C}: \mathrm{H}$ IR spectrum of the a-C:H produced is presented on the right panel of Fig. 1.

The infrared transmission spectrum and its absorption bands give us access to the structure of the a-C:H produced. Table 1 presents the assignment of the prominent bands observed in the film spectra (e.g. Ristein et al. 1998; Dartois et al. 2004, 2005). Interference fringes appear in the infrared transmission spectra, resulting from the multiple reflections at the film interfaces. These fringes can give us information about the thickness and the optical constants of the film necessary to the PL study. In the region devoid of absorption, the interfringe $\Delta \sigma\left(\mathrm{in} \mathrm{cm}^{-1}\right)$ is a function of the sample thickness $H$ and of its refraction index $n_{0}$ :

$\Delta \sigma=\frac{1}{2 H n_{0}}$

Alternatively, for thin films, the $3.4 \mu \mathrm{m}$ band absorbance is used to scale the film thickness to previous depositions under the same conditions.

\subsubsection{UV-visible spectroscopy}

The UV-visible transmission spectra of a-C:H film deposited onto a quartz substrate (see left of Fig. 1) are measured in the spectral range between $210 \mathrm{~nm}$ and $710 \mathrm{~nm}$ with a $2 \mathrm{~nm}$ resolution, using an Avalight DS-DUV deuterium lamp and a fibered Avaspec-2048 Czerny-Turner grating spectrometer.

With this transmission spectrum and the film thickness obtained with the IR spectrum, we can establish a first-order UV-visible imaginary part $\kappa(\lambda)$ of the a-C:H complex index or the film absorption coefficient $\alpha(\lambda)$ in this range. These parameters are linked to the transmittance $T(\lambda)$ measurements by Eq. (2) and are needed to evaluate the PL yield: $\alpha(\lambda)$ will allow us to evaluate how the incident excitation (giving rise to the photoluminescence) is absorbed by the sample and how it is distributed in the film:

$T(\lambda)=\exp [-\alpha(\lambda) H]=\exp \left[-\frac{4 \pi}{\lambda} \kappa(\lambda) H\right]$.

We can then determine the a-C:H optical gap, often defined in the literature by $E_{04}$, i.e., the energy where the absorption coefficient is $10^{4} \mathrm{~cm}^{-1}\left(\alpha\left(E_{04}\right)=10^{4} \mathrm{~cm}^{-1}\right)$. The a-C:H gaps are sometimes also defined as the Tauc gap $E_{\text {Tauc }}$. The quantity $\sqrt{\alpha E}$ is proportional to the energy difference $E-E_{\text {Tauc }}, E_{\text {Tauc }}$ is therefore obtained by plotting $\sqrt{\alpha E}$ versus $E$. It has been observed that $E_{04}$ exceeds $E_{\text {Tauc }}$ by an approximatively constant energy of $0.6 \mathrm{eV}$ (e.g. Silva et al. 1996). In this paper, we use $E_{04}$.

\subsubsection{Refractive index determination}

The real part of the sample refractive index $n_{0}$ is estimated from the sample optical gap $E_{04}$ because these both parameters are correlated. We brought together in Fig. 2 a-C:H refractive indexes and optical gaps data from different studies (see references in caption). When the optical gap is given by $E_{\text {Tauc }}$ (red point in Fig. 2), we add $0.6 \mathrm{eV}$ to infer the $E_{04}$ gap. This well seen correlation between $n_{0}$ and $E_{04}$ gives refractive indexes between 1.25 and 1.85 for our a-C:H samples.

Information about $n_{0}$ can also be found in the interferences pattern of the IR transmission spectra (Swanepoel 1983). We compared the refractive index estimated for few a-C:H samples from the IR spectra obtained by micro-spectroscopy to the one found from the optical gap, and a good agreement exists between these methods. 


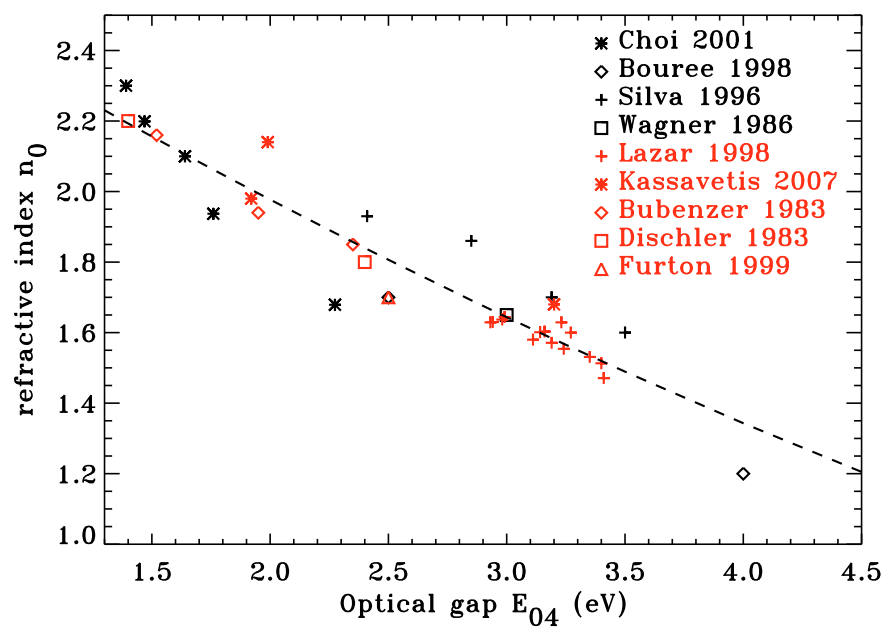

Fig. 2. Refractive index of different a-C:H samples as a function of the optical gap (obtained by Bubenzer et al. 1983; Dischler et al. 1983; Wagner \& Lautenschlager 1986; Silva et al. 1996; Bourée et al. 1998; Lazar 1998; Furton et al. 1999; Choi 2001; and Kassavetis et al. 2007). For data in red, only the $E_{\text {Tauc }}$ gap was given, so we add $0.6 \mathrm{eV}$ to obtain approximatively the $E_{04}$ gap. The dashed line is a fit to the data.

\subsection{4. $\mathrm{sp}^{2} / \mathrm{sp}^{3}$ ratio determination}

Carbon atoms in a-C:H exist in both $\mathrm{sp}^{2}$ and $\mathrm{sp}^{3}$ electronic configurations. The $\pi$ and $\pi^{*}$ states of the $\mathrm{sp}^{2}$ sites control the optical gap. A correlation exists between the fraction of carbons in the $\mathrm{sp}^{2}$ configuration and the optical gap. Figure 3 presents results from different workers who measured these two parameters for a-C:H samples (Jarman et al. 1986; Kleber 1991; Tamor \& Vassell 1994; Robertson 1996; Kassavetis et al. 2007). The Dartois et al. (2005) a-C:H sample is represented by the colored area: the sample optical gap is evaluated from its high degree of hydrogenation and low refractive index. The $\mathrm{sp}^{2}$ content is estimated between $5 \%$ and $20 \%$ (with $20 \%$ a tight upper limit) from IR spectrum. In addition to a-C:H samples, extremes cases of glassy carbon, graphite (all $\mathrm{sp}^{2}$ ), and diamond (all $\mathrm{sp}^{3}$ ) are also represented. The fitted $\mathrm{sp}^{2}$ fraction is linked to the Tauc gap by the following relation:

$$
\begin{aligned}
\frac{\mathrm{sp}^{2}}{\mathrm{sp}^{2}+\mathrm{sp}^{3}}= & 0.99( \pm 0.04)-0.38( \pm 0.03) E_{\text {Tauc }} \\
& +0.036( \pm 0.006) E_{\text {Tauc }}^{2} .
\end{aligned}
$$

To obtain information about the structure of our a-C:H materials, we estimated the $\mathrm{sp}^{2}$ fraction from this relation and the optical gaps we measured. For our samples, $\mathrm{sp}^{2} / \mathrm{sp}^{3}$ ratios are found between $5 \%$ and $30 \%$.

\subsection{Photoluminescence measurements}

To quantitatively measure the photoluminescence of our produced films, we have at our disposal a double monochromator (for excitation and emission) Perkin Elmer LS55 luminescence spectrometer coupled to a front surface device. It allows us to measure the photoluminescence of the a-C:H between $200 \mathrm{~nm}$ and $800 \mathrm{~nm}$. The photoluminescence of the sample is measured ex-situ at room temperature just after its production. The external medium during this measurement, designed by the subscript 1 , is the air. The quasi-monochromatic excitation (excitation $F W H M<10 \mathrm{~nm}$ ) of the samples occurs at UV-visible wavelengths greater than $200 \mathrm{~nm}$ but quantitative measurements are

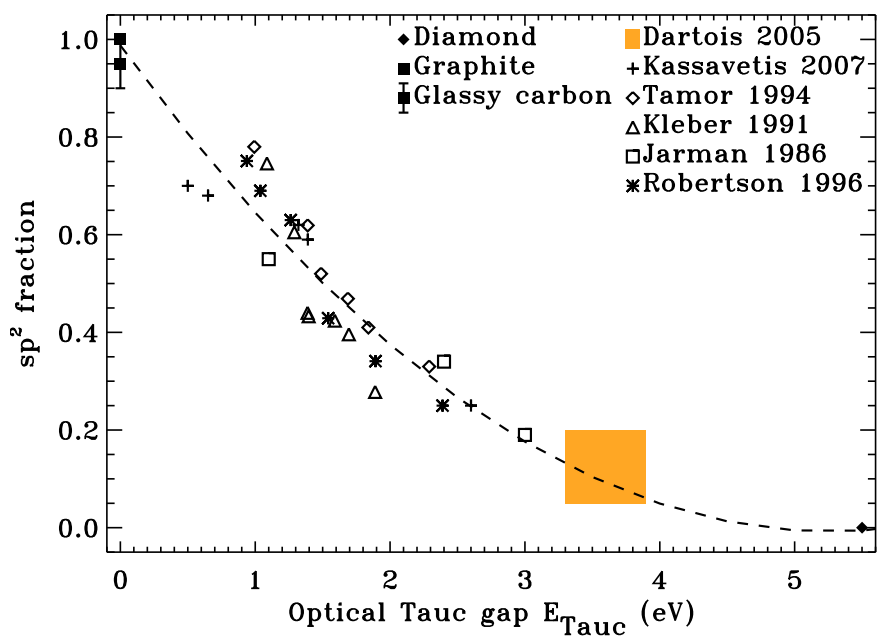

Fig. 3. $\mathrm{sp}^{2}$ fraction of different a-C:H samples as a function of the Tauc optical gap (obtained by Jarman et al. 1986; Kleber 1991; Tamor \& Vassell 1994; Robertson 1996; and Kassavetis et al. 2007). The colored area corresponds to the Dartois et al. (2005) a-C:H sample (see text for details). Extreme cases of glassy carbon (Robertson 1986; Turlo \& Rozwadowskajasniewska 1987), graphite (all $\mathrm{sp}^{2}$ ) (filled squares), and diamond (all $\mathrm{sp}^{3}$ ) (filled diamond) are also represented. The dashed line is a second-degree polynomial fit to these data.

possible for excitation wavelength greater than $250 \mathrm{~nm}(\sim 5 \mathrm{eV})$, using the xenon flash lamp of the LS55. The excitation angle $\alpha_{\text {exc, } 1}$ and the emission detection angle $\alpha_{1}$ (from the sample surface normal) are performed at 30 and -60 degrees, respectively, to get rid of any specular reflexion. We use almost perfect Lambertian reflectance standards (Labsphere Spectralon diffuse reflectance standards) as reference to measure the incident excitation flux at the location of the sample as well as the relative instrument response.

For absolute calibration purposes, we replace the front assay device by a Labsphere integrating sphere coupled to the LS55 luminescence spectrometer with optic fibers in order to provide a second and independent measurement of absolute PL yield. This set up was used to build our own PL standard to check the validity of our absolute quantum yield calculation (explained in the next section). A bismuth germanate crystal $\left(\mathrm{Bi}_{4} \mathrm{Ge}_{3} \mathrm{O}_{12}\right.$, BGO hereafter) window of the same shape as our substrate was used as PL standard. BGO PL yield is high, remains stable, and can be easily manipulated. Moreover, because of its high Stokes' shift, there is no overlap of the absorption and emission spectra (re-absorption of the emission by the sample being not easily considered when using an integrating sphere).

\section{Determination of the absolute intrinsic photoluminescence quantum yield}

\subsection{With the luminescence spectrometer}

The intrinsic quantum yield determination requires perfect knowledge of the optical effects implied in the measurement. The detailed calculations are presented in Appendices A and B, and we summarize here the steps needed to retrieve this yield. In the appendixes, the emission of light from a thin film is modeled and the interference effects of the reflections on the interfaces of the film are calculated (Lukosz 1981; Holm et al. 1982; Nollau et al. 2000). The thin film is an absorbing dielectric layer (the subscript 0 is used to design this medium) located between two different media 1 (the external medium) and 2 
(the substrate). The luminescent centers are assumed to be electric dipole sources with randomly oriented dipole moment $\boldsymbol{p}$.

We consider the light transmitted outside the film (i.e. in medium 1) separated into two beams: the direct beam $D$ is the one emitted directly in the direction of medium 1 toward the detector, and the reflected beam $R$ is the beam that is first directed toward the substrate (i.e. medium 2) and reflected off the back surface of the film (i.e. interface $0 / 2$ ) before being transmitted in medium 1. The emission angles in the film of the $D$ and $R$ beams are thus $\alpha_{0}$ and $\pi-\alpha_{0}$, respectively, from the sample surface normal (figures of this geometry exist in the appendixes). The angle $\alpha_{0}$ is given by the observation angle in medium $1, \alpha_{1}$, and the Snell-Descartes refraction law. Each of these beams is reflected many times between each interface of the film and will influence the measured yield with respect to the intrinsic one.

The expressions of these beams amplitude account for the eventual self-absorption of the emission by the film from the emitting dipole location to medium 1 (considered nonabsorbing), calculated from the absorption coefficient (the selfabsorption being expressed by $\mathrm{e}^{-\tau_{0}(h)}$ in relation 6).

Both of these beams undergo losses when they are transmitted out of the film or reflected inside the film. These losses are expressed through the Fresnel coefficients, $t_{i j}^{(\mathrm{s}, \mathrm{p})}$ and $r_{i j}^{(\mathrm{s}, \mathrm{p})}$, expressed for complex indexes in Appendix C. For example, the transmittance of the emitted power at the interface between a-C:H material and air is

$T_{i j}^{(\mathrm{s}, \mathrm{p})}=\frac{n_{j} \cos \alpha_{j}}{n_{i} \cos \alpha_{i}}\left\|t_{i j}^{(\mathrm{s}, \mathrm{p})}\right\|^{2}$.

There is also a significant modification of the radiant intensity between media 0 and 1 because of the modification at the interface of the solid angle under which the emission is detected. This modification is expressed by the ratio of infinitesimal solid angles in each medium (Appendix B):

$\frac{\mathrm{d} \Omega_{0}}{\mathrm{~d} \Omega_{1}}=\frac{n_{1}^{2} \cos \alpha_{1}}{n_{0}^{2} \cos \alpha_{0}}$.

The reflections at the film interfaces of each beam create an interference effect called the multiple-reflections interference effect. The superposition of the $D$ and $R$ beams (whose directions of emission form an angle equals to $\pi-2 \alpha_{0}$ ) gives rise to the wide-angle interference effect. These effects are represented by $M$ and $W$ calculated in Appendix B.

The emitted photoluminescence power is proportional to the power of absorbed light and to the photoluminescence efficiency $\eta_{\mathrm{E}}$. The power absorbed by the film at the depth $h$ on a thickness $\mathrm{d} h$ is $\operatorname{Abs}(h) \mathrm{d} h$ and the radiant intensity (in $\mathrm{W} / \mathrm{sr}$ ) emitted by this film thickness is then $\eta_{\mathrm{E}} \frac{\operatorname{Abs}(h) \mathrm{d} h}{4 \pi}$. The resulting emission of this monochromatic absorption is shared out on a range of visible wavelengths according to a normalized $^{2}$ emission profile $\operatorname{Em}(\lambda)$. The determination of the absorption profile Abs (as a function of the film depth $h$ and of the excitation wavelength $\lambda_{\text {exc }}$ ) results from the absorption coefficient $\alpha(\lambda)$ calculated from the UV-visible transmission spectrum (see Sect. 2.2.2) and the incident excitation power on the sample $P_{\text {incident }}$ :

$\operatorname{Abs}(h) \mathrm{d} h=-\mathrm{d}\left(T_{10}\left(\lambda_{\text {exc }}\right) \exp \left[-\frac{\alpha\left(\lambda_{\text {exc }}\right) h}{\cos \alpha_{\text {exc }, 0}}\right] P_{\text {incident }}\right)$

$\operatorname{Abs}(h) \mathrm{d} h=T_{10}\left(\lambda_{\mathrm{exc}}\right) \frac{\alpha\left(\lambda_{\mathrm{exc}}\right) \mathrm{d} h}{\cos \alpha_{\mathrm{exc}, 0}} \exp \left[-\frac{\alpha\left(\lambda_{\mathrm{exc}}\right) h}{\cos \alpha_{\text {exc }, 0}}\right] P_{\text {incident }} .(5)$

$2 \int \operatorname{Em}(\lambda) \mathrm{d} \lambda=1$
A complete calculation done in the appendixes shows that the specific radiant intensity $I_{1}(\lambda)$ (in $\mathrm{W} \mathrm{sr}^{-1} \mathrm{~nm}^{-1}$ ), emitted in medium 1 at wavelength $\lambda$ and in the direction fixed by $\alpha_{0}$, the emission angle in the film, is expressed by the following equation (the (s) and (p) polarizations of the light are distinguished by the corresponding superscripts ${ }^{3}$ ):

$I_{1}(\lambda)=I_{1}^{(\mathrm{s})}(\lambda)+I_{1}^{(\mathrm{p})}(\lambda)$

$I_{1}^{(\mathrm{s}, \mathrm{p})}(\lambda)=\int_{0}^{H} \mathrm{e}^{-\tau_{0}(h)} T_{01}^{(\mathrm{s}, \mathrm{p})} \frac{\mathrm{d} \Omega_{0}}{\mathrm{~d} \Omega_{1}} M^{(\mathrm{s}, \mathrm{p})} W^{(\mathrm{s}, \mathrm{p})} \frac{\eta_{\mathrm{E}} \operatorname{Em}(\lambda) \operatorname{Abs}(h) \mathrm{d} h}{8 \pi}$

where

$M^{(\mathrm{s}, \mathrm{p})}=1+\rho_{01}^{(\mathrm{s}, \mathrm{p}) 2} \rho_{02}^{(\mathrm{s}, \mathrm{p}) 2} \mathrm{e}^{-2 \tau_{0}(H)}-2 \rho_{01}^{(\mathrm{s}, \mathrm{p})} \rho_{02}^{(\mathrm{s}, \mathrm{p})} \mathrm{e}^{-\tau_{0}(H)} \cos \left(\Delta^{(\mathrm{s}, \mathrm{p})}\right)$

$W^{(\mathrm{s})}=1+\rho_{02}^{(\mathrm{s}, \mathrm{p}) 2} \mathrm{e}^{-\tau_{w}}+2 \rho_{02}^{(\mathrm{s}, \mathrm{p})} \mathrm{e}^{-\tau_{w} / 2} \cos \left(\delta_{w}^{(\mathrm{s})}\right)$

$W^{(\mathrm{p})}=1+\rho_{02}^{(\mathrm{s}, \mathrm{p}) 2} \mathrm{e}^{-\tau_{w}}+2 \rho_{02}^{(\mathrm{s}, \mathrm{p})} \mathrm{e}^{-\tau_{w} / 2} \cos \left(\delta_{w}^{(\mathrm{p})}\right) \cos \left(2 \alpha_{0}\right)$

$\tau_{0}(h)=\frac{4 \pi}{\lambda} \kappa_{0} \frac{h}{\cos \alpha_{0}}$

$\tau_{w}=\frac{8 \pi}{\lambda} \kappa_{0} \frac{H-h}{\cos \alpha_{0}}$

$\Delta^{(\mathrm{s}, \mathrm{p})}=\frac{4 \pi}{\lambda} n_{0} H \cos \alpha_{0}+\delta_{01}^{(\mathrm{s}, \mathrm{p})}+\delta_{02}^{(\mathrm{s}, \mathrm{p})}$

$\delta_{w}^{(\mathrm{s}, \mathrm{p})}=\frac{4 \pi}{\lambda} n_{0}(H-h) \cos \alpha_{0}+\delta_{02}^{(\mathrm{s}, \mathrm{p})}$,

where $n_{i}$ and $\kappa_{i}$ are respectively the real and imaginary part of the medium $i$ index, $\rho_{i j}^{(\mathrm{s}, \mathrm{p})}$ and $\delta_{i j}^{(\mathrm{s}, \mathrm{p})}$ are the modulus and argument of Fresnel reflection coefficients, and $T_{i j}^{(\mathrm{s}, \mathrm{p})}$ is the Fresnel transmittance at the interface $i / j$. They are expressed in Appendix C.

The emitted specific power detected by the LS55 instrument in the solid angle $\Omega_{1}$ (after correction by the instrument response) is

$P_{1}(\lambda)=\int_{\Omega_{1}} I_{1}(\lambda) \mathrm{d} \Omega$.

With $I_{1}(\lambda)$ varying little on the small detection solid angle $\Omega_{1}$, we can consider that: $P_{1}(\lambda)=I_{1}(\lambda) \cdot \Omega_{1}$.

The photoluminescence efficiency $\eta_{\mathrm{E}}$, defined as a ratio of energy or power, is then obtained:

$\eta_{\mathrm{E}}=\int_{\lambda} \frac{P_{1}(\lambda)}{\Omega_{1}} \frac{\mathrm{d} \Omega_{1}}{\mathrm{~d} \Omega_{0}} \frac{1}{S_{\mathrm{E}}} \mathrm{d} \lambda$,

where

$$
\begin{aligned}
S_{\mathrm{E}}= & \int_{0}^{H}\left(T_{01}^{(\mathrm{s})}(\lambda) M^{(\mathrm{s})}(\lambda) W^{(\mathrm{s})}(\lambda, h)+T_{01}^{(\mathrm{p})}(\lambda) M^{(\mathrm{p})}(\lambda) W^{(\mathrm{p})}(\lambda, h)\right) \\
& \times \mathrm{e}^{-\tau_{0}(h)} \frac{1}{4 \pi} \frac{\operatorname{Abs}(h)}{2} \mathrm{~d} h .
\end{aligned}
$$

In most astrophysical papers about the extended red emission, the yield of this observed emission feature is given as a quantum

\footnotetext{
3 We consider that the emission in the film is equally split up into the two polarizations. Radiant intensities polarized $\mathrm{s}$ and $\mathrm{p}$ emitted in the film are $I_{0}^{(\mathrm{s})}=I_{0}^{(\mathrm{p})}=\frac{1}{2} \eta_{\mathrm{E}} \frac{\mathrm{Abs}(h) \mathrm{d} h}{4 \pi}$.
} 
yield, i.e., a ratio of photons numbers, whereas we measure the energy. To do this conversion, the energy has to be multiplied by $\frac{\lambda}{h c}$. The quantum yield is thus obtained by Eq. (9):

$$
\begin{aligned}
S= & \int_{0}^{H}\left(T_{01}^{(\mathrm{s})}(\lambda) M^{(\mathrm{s})}(\lambda) W^{(\mathrm{s})}(\lambda, h)+T_{01}^{(\mathrm{p})}(\lambda) M^{(\mathrm{p})}(\lambda) W^{(\mathrm{p})}(\lambda, h)\right) \\
& \times \mathrm{e}^{-\tau_{0}(h)} \lambda_{\operatorname{exc}} \frac{1}{4 \pi} \frac{\operatorname{Abs}(h)}{2} \mathrm{~d} h
\end{aligned}
$$$$
\eta=\int_{\lambda} \lambda \frac{P_{1}(\lambda)}{\Omega_{1}} \frac{\mathrm{d} \Omega_{1}}{\mathrm{~d} \Omega_{0}} \frac{1}{S} \mathrm{~d} \lambda .
$$

Taking all these optical effects into account allows us to obtain the intrinsic and absolute photoluminescence quantum yield of our a-C:H material, instead of an external yield that can appear significantly lower than the intrinsic efficiency when these effects are neglected.

The variable $P_{1}(\lambda)$ is obtained from the power detected by the instrument $P_{1, \mathrm{LS} 55}(\lambda)$ corrected by $R_{\mathrm{LS} 55}(\lambda)$, the instrument response between the sample and the detector: $P_{1}(\lambda)=$ $P_{1, \mathrm{LS} 55}(\lambda) / R_{\mathrm{LS} 55}(\lambda)$. A Labsphere Spectralon Lambertian reflectance standard used at the sample location allows us to measure the true incident excitation $P_{\text {incident }}$. The reflectance $\mathcal{R}(\lambda)$ of this standard is known. The radiant intensity diffused by the Lambertian standard in the direction given by $\alpha_{1}$ from the surface normal is $P_{\text {incident }} / \pi \cdot \cos \alpha_{1} \cdot \mathcal{R}$. Thus, $P_{\text {incident }}$ is linked to the measurement $P_{\text {incident,LS55 }}$ by the relation:

$P_{\text {incident }}=\frac{P_{\text {incident } \mathrm{LS} 55}}{R_{\mathrm{LS55}}} \cdot \frac{1}{\mathcal{R}} \cdot \frac{\pi}{\int_{\Omega_{1}} \cos \alpha_{1} \mathrm{~d} \Omega}$.

It should be noted that, in this description allowing us to calculate the PL efficiency, we do not take the secondary PL emission, i.e., the PL emission by the sample following the selfabsorption of a previous PL photon, into account. We only take the self-absorption into account, not the eventual additional PL that could result of this self-absorption. As pointed out by Malloci et al. (2004), the contribution of this additional emission is very small. Moreover, this secondary photoluminescence can occur only when there is a superposition of the absorption and emission bands. This superposition is weak for our samples. Therefore, the obtained PL yields when neglecting the secondary PL are not significantly overestimated.

\subsection{With the integrating sphere: validation of the absolute yield determination}

To check the validity of the model described above and used to determine the PL absolute yields of our a-C:H samples, we determined the PL absolute quantum yield of a BGO sample by two independent methods. The first one is the method described in Sect. 3.1 and also used for a-C:H samples (media 0 and 2 being both BGO as there is no substrate for this sample), and the other one results from a measurement with an integrating sphere (using the excitation and the detection blocks of the luminescence spectrometer). The PL absolute yield when using an integrating sphere is determined with the method given in De Mello et al. (1997). The results found by both methods, presented in Fig. 4, are in good agreement. These absolute values are also similar to the one found in Rodnyi (1997) and Weber \& Monchamp (1973) $(\eta \approx 13 \%$ at $295 \mathrm{~K}$ ). This validates our method for calculating absolute internal yield from film photoluminescence measurement with the luminescence spectrometer.

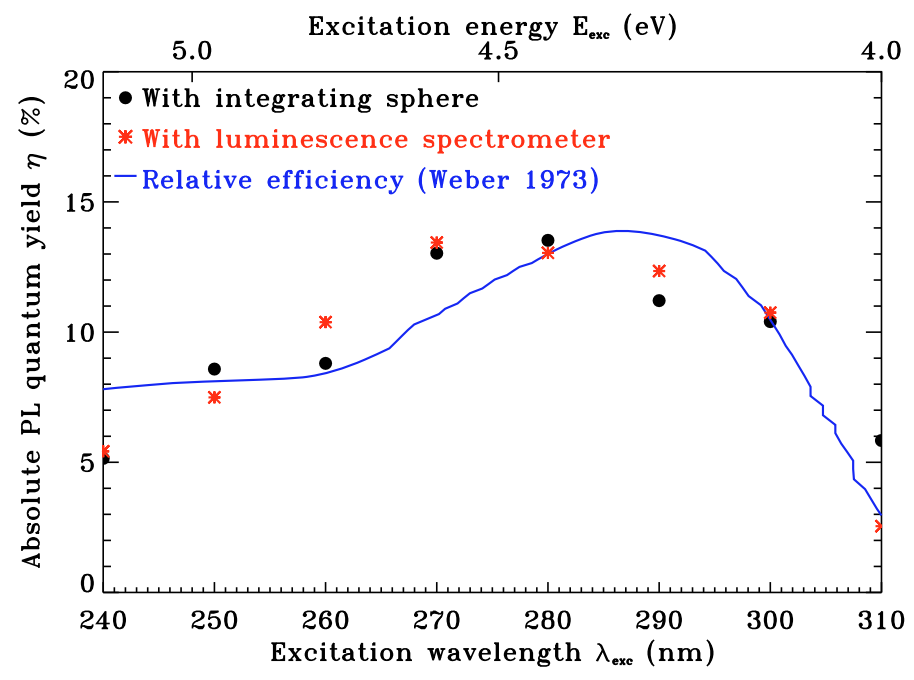

Fig. 4. BGO absolute PL quantum yield for different excitation wavelengths, determined by two independent methods: with a front surface device (crosses) or with an integrating sphere (circles). The variation with excitation of PL intensity (i.e. relative efficiencies) found in Weber \& Monchamp (1973) is also displayed for comparison (solid line).

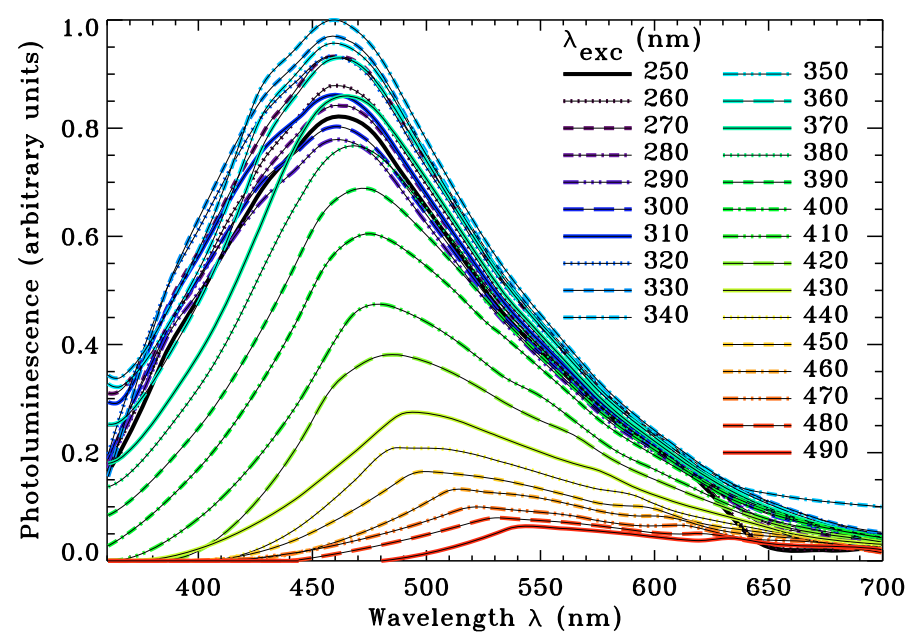

Fig. 5. Examples of the photoluminescence spectra of an a-C:H sample, for different excitation wavelengths $\left(\lambda_{\mathrm{exc}} \in[250,490] \mathrm{nm}\right)$.

\section{Results}

The produced a-C:H samples are yellow orange films (at visible light), with a thickness varying typically from 0.5 to 10 microns. The photoluminescence of these films seen under an UV lamp (optical engineering's model 22-UV) has an apparent color varying from yellow to red. We display in Fig. 5 an example of an a-C:H sample photoluminescence spectrum measured for different excitation wavelengths ranging from 250 to $490 \mathrm{~nm}$. The PL color of this sample under the UV lamp appears yellow to the eye. The photoluminescence is a broad and featureless band. The PL band does not seem to shift with varying excitation energy except when the excitation occurs for wavelengths corresponding to those of the PL band: in such cases, the PL band moves to greater wavelengths (since $E_{\mathrm{PL}} \leq E_{\mathrm{exc}}$ ) and its intensity decreases until the emission does not occur at all because the excitation goes out of the absorption band (as seen in Fig. 1). 
M. Godard and E. Dartois: Photoluminescence of hydrogenated amorphous carbons

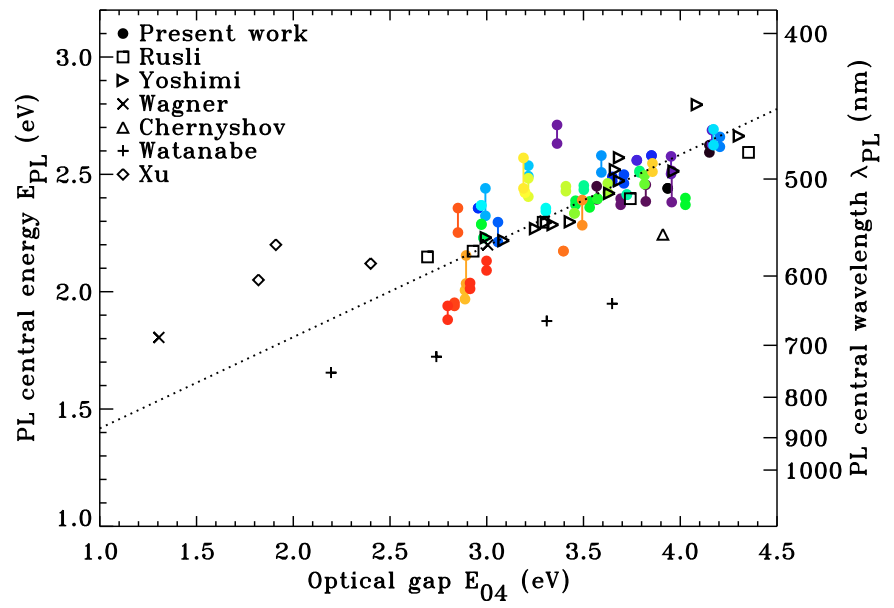

Fig. 6. Variation in the a-C:H photoluminescence color with the material optical gap $E_{04}$. Our a-C:H samples (filled colored circles, each color corresponding to an a-C:H sample) are plotted with other a-C:H photoluminescence studies (other black symbols) for comparison (Watanabe et al. 1982; Wagner \& Lautenschlager 1986; Chernyshov et al. 1991; Yoshimi et al. 1992; Xu et al. 1993; Rusli et al. 1996). The dotted line is a fit to the data.

We produced samples of different a-C:H types depending on the production conditions. The properties of the films are mainly determined by the impact energy of the ions/radicals, which is controlled by the power applied and the chamber gas pressure. The a-C:H materials were characterized by their optical gap $E_{04}$, ranging from 2.8 to $4.3 \mathrm{eV}$. Material differences, represented by $E_{04}$ variations, induce differences in the photoluminescence: Fig. 6 shows that the photoluminescence band moves to longer wavelengths when the optical gap decreases (PL appears yellow under UV lamp for a-C:H with gap around $4 \mathrm{eV}$ and red for $3 \mathrm{eV}$ gap a-C:H), in agreement with results from other groups also displayed on this figure (see references in caption).

The PL color changes with the optical gap of the material, but also the PL efficiency. In Fig. 7, we can see that the absolute quantum yield of our a-C:H samples decreases from about $3 \%$ to $0.01 \%$ when $E_{04}$ varies from 4.3 to $2.8 \mathrm{eV}$. Previous studies from other groups (see references in caption) give a-C:H PL relative efficiency results and the exponential decrease of the relative efficiency with the optical gap agrees with our results. We converted these relative quantum yields into absolute ones by adjusting the whole of them to our absolute values. Then, a-C:H PL absolute yields are obtained on a wider range of optical gaps than with only our samples. The exponential fit of these data gives an absolute quantum yield variation with the $E_{04}$ optical gap (in eV) with the following equation (the $3 \sigma$ uncertainty estimation for the fit parameters are indicated and corresponds to the dotted lines in Fig. 7):

$\eta=0.44( \pm 0.39) \cdot \exp \left(\frac{-16( \pm 2)}{E_{04}-0.6}\right)$.

The PL quantum yield can also be linked to the estimated $\mathrm{sp}^{2}$ fraction as shown in Fig. 8. Figures 7 and 8 show exponential fits that correspond to an equation of the form $\eta=\eta_{0} \exp [-K /(1-$ $\left.\left.\frac{\mathrm{sp}^{2}}{\mathrm{sp}^{2}+\mathrm{sp}^{3}}\right)\right]$, given by Robertson (1996). The denominator $E_{04}-0.6$ in the exponential of Eq. (11) fits well the data (see the fit in Fig. 7) and is coherent with both the shift between $E_{04}$ and $E_{\text {Tauc }}$ and the variation in $\mathrm{sp}^{2}$ fraction with the optical gap: an $\mathrm{sp}^{2}$ fraction value of 1 corresponds to an $E_{\text {Tauc }}$ value around 0 , hence

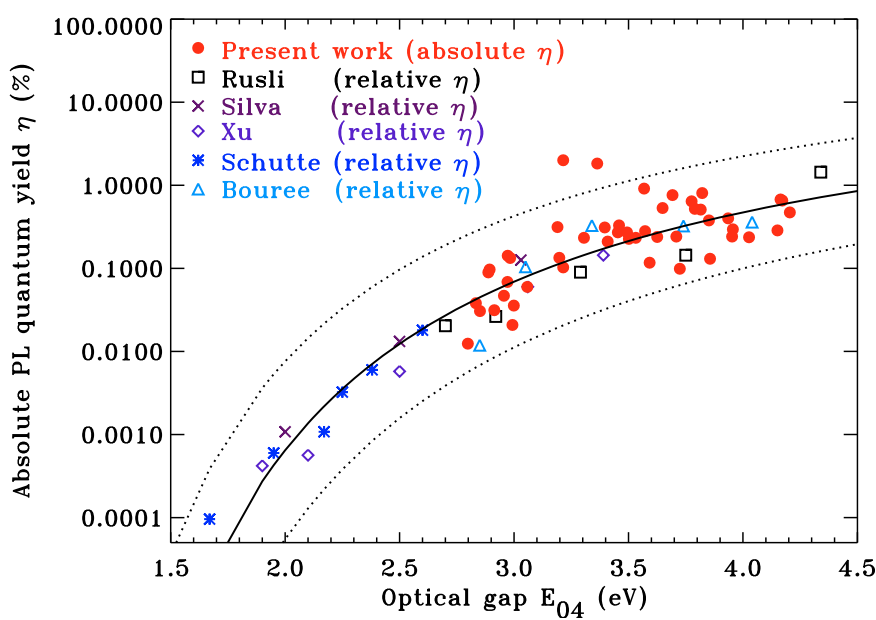

Fig. 7. Variation in the a-C:H absolute photoluminescence quantum yield $\eta$ with the a-C:H optical gap $E_{04}$. Relative quantum yields result from other photoluminescence studies (Schütte et al. 1993; Xu et al. 1993; Silva et al. 1996; Rusli et al. 1996; Bourée et al. 1998) are converted into absolute quantum yields using our absolute results (filled red circles) and added to this figure. The solid line is an exponential fit to all these efficiency data (see text for details) and each of the dotted lines corresponds to a $3 \sigma$ variation in the fit parameters.

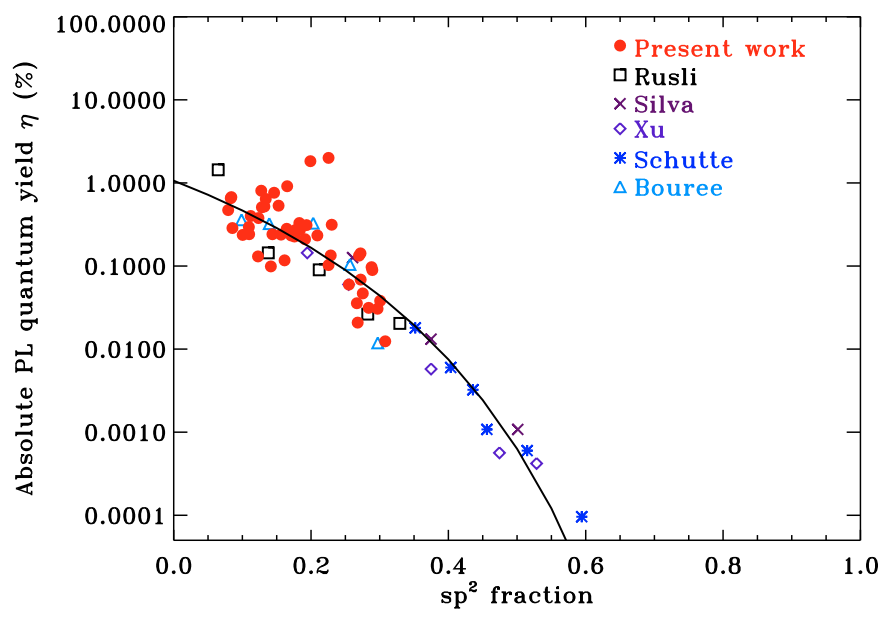

Fig. 8. Variation in the a-C:H absolute photoluminescence quantum yield $\eta$ with the estimated $\mathrm{sp}^{2}$ fraction (from Fig. 3). The solid line is an exponential fit to the data.

to $E_{04}$ value around 0.6. The denominator $1-\frac{\mathrm{sp}^{2}}{\mathrm{sp}^{2}+\mathrm{sp}^{3}}$ becomes $E_{04}-0.6$. They described the PL mechanism in a-C:H by extending a model proposed for a-Si:H PL, i.e., with PL arising from the radiative recombination of electrons and holes trapped in band tail states and described by a one electron model. Even if it seems to agree with experimental data, the work of Heitz et al. (1999) points out that the a-C:H PL properties indicate a strong electron-hole Coulomb interactions and suggest an exciton-like behavior. The PL exponential dependence observed for a-C:H may be governed by exciton electron-hole pair dissociation as the photogenerated electron tunnels to an acceptor site, whose density increases when the optical gap decreases.

With Figs. 6 and 7 together, we can deduce that the PL yield decreases when the photoluminescence central wavelength increases. This is shown in Fig. 9: a-C:H emission varies from yellow highly efficient PL to red and lower efficient PL when the gap decreases. This corresponds to a PL central wavelength range between 460 and $660 \mathrm{~nm}$. One can see in Fig. 10 that the 


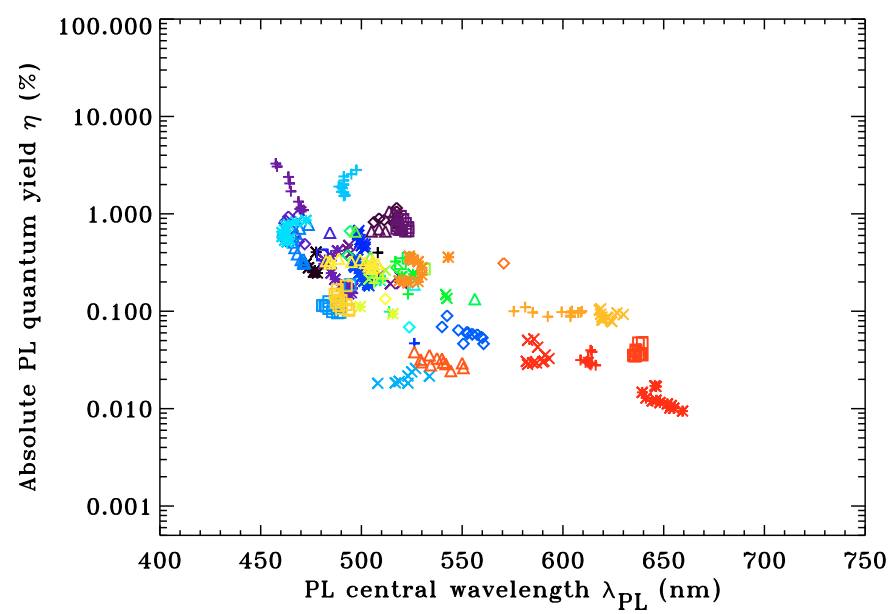

Fig. 9. Variation in the a-C:H photoluminescence quantum yield $\eta$ with the photoluminescence central wavelength. Different produced a-C:H samples are represented with different symbols and colors (the different points for one sample correspond to the different excitation wavelengths between 250 and $370 \mathrm{~nm}$ ).

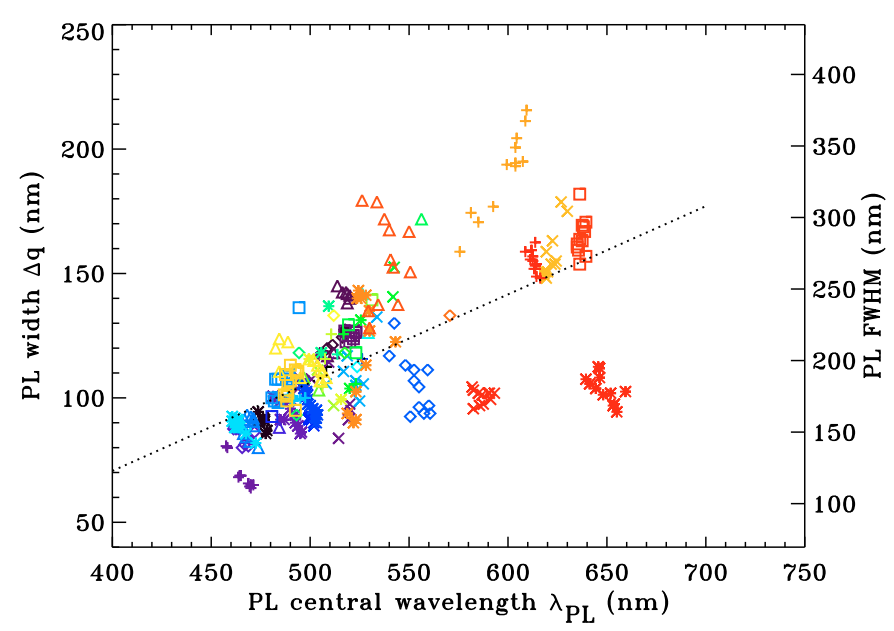

Fig. 10. Variation in the a-C:H photoluminescence band width $\Delta q$ (the correspondence with $F W H M$ is shown by the right axis) with the central wavelength of the photoluminescence band (i.e., the photoluminescence color). Different a-C:H samples produced are represented with different symbols and colors. Only measurements with $\lambda_{\text {exc }} \leq 370 \mathrm{~nm}$ are plotted. The dotted line is a fit to the data.

width of the band $\Delta q$ is correlated to the PL color and increases from about 60 to $210 \mathrm{~nm}$ (i.e $F W H M \in[110,370] \mathrm{nm}$ ) when the band moves to longer wavelengths.

The influence of the excitation wavelength on the a-C:H photoluminescence is shown in Figs. 11 and 12. In Fig. 11, the PL central wavelength is plotted as a function of the excitation wavelength for different samples. The PL band position does not vary with $\lambda_{\text {exc }}$, except when the excitation occurs in the PL band (i.e., to the right of the dotted line) since the emission energy is necessarily lower than the excitation energy. When this situation happens, light is emitted only in the high-wavelength part of the PL band, and the PL central wavelength decays to a redder color as observed in Figs. 11 and 5.

In the explored range, the excitation energy does not seem to have a strong influence on the PL color but also on the PL efficiency as shown in Fig. 12. The absolute quantum yield as a function of the excitation wavelength is almost constant.

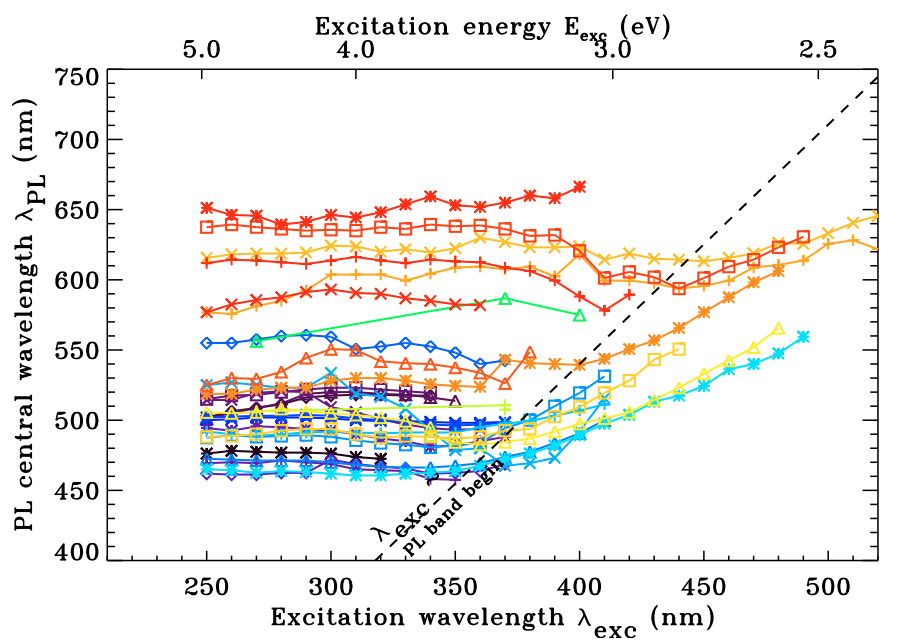

Fig. 11. Variation in the a-C:H photoluminescence central wavelength with the excitation wavelength for different a-C:H samples, represented with different colors and symbols linked by lines as guides for the eye. The dashed line represents the equation $\lambda_{\mathrm{exc}}=\lambda_{\mathrm{PL}}-\frac{2}{3} F W H M$ (the relation between $\lambda_{\mathrm{PL}}$ and $F W H M$ determined from the Fig. $10 \mathrm{fit}$ is used). Thus, this line represents the beginning of the photoluminescence band and, in the area to the right of this line, the excitation occurs in the PL band.

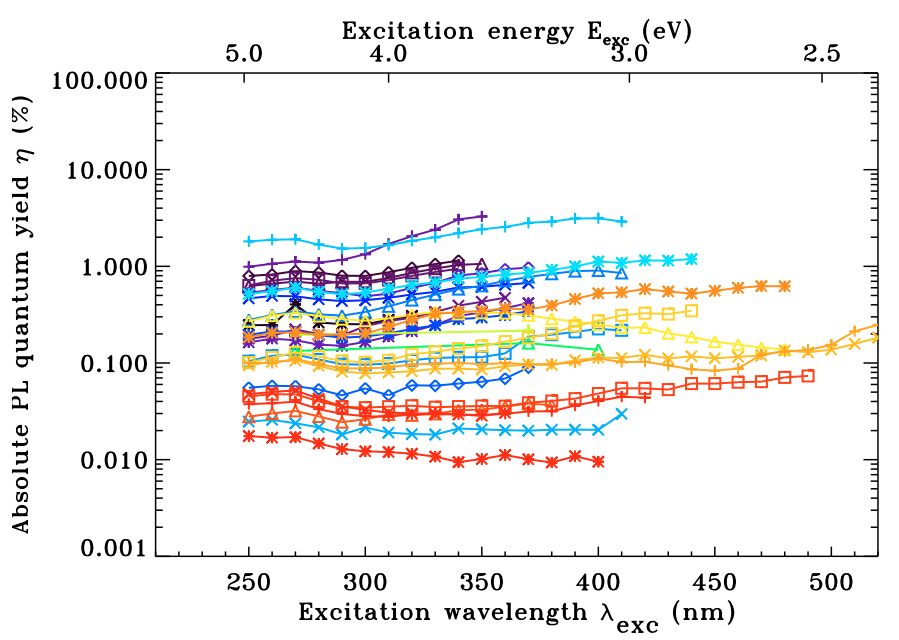

Fig. 12. Variation in the a-C:H photoluminescence yield with the excitation wavelength for different a-C:H samples, represented with different colors and symbols linked by lines as guides for the eye.

\section{Discussion}

\subsection{Photoluminescence spectra}

To compare the photoluminescence of our a-C:H with extended red emission observations, we display the PL width $\Delta q$ (and the $F W H M)$ in Fig. 13 as a function of the emission peak wavelength $\lambda_{\mathrm{PL}}$, of both a-C:H laboratory samples and ERE observations. The a-C:H PL data are those presented in Fig. 10 of this paper, together with data from Lin \& Feldman (1982), Lin \& Feldman (1983), Watanabe et al. (1982), Wagner \& Lautenschlager (1986), Chernyshov et al. (1991), Xu et al. (1993), Rusli et al. (1996), Ledoux et al. (2001), and Dartois et al. (2005). The ERE observations data are taken from Fig. 1 in Darbon et al. (1999), which regrouped most of the ERE spectral observations. We also add the ERE observations corresponding to the DISM (Szomoru \& Guhathakurta 1998) and L780 dark 


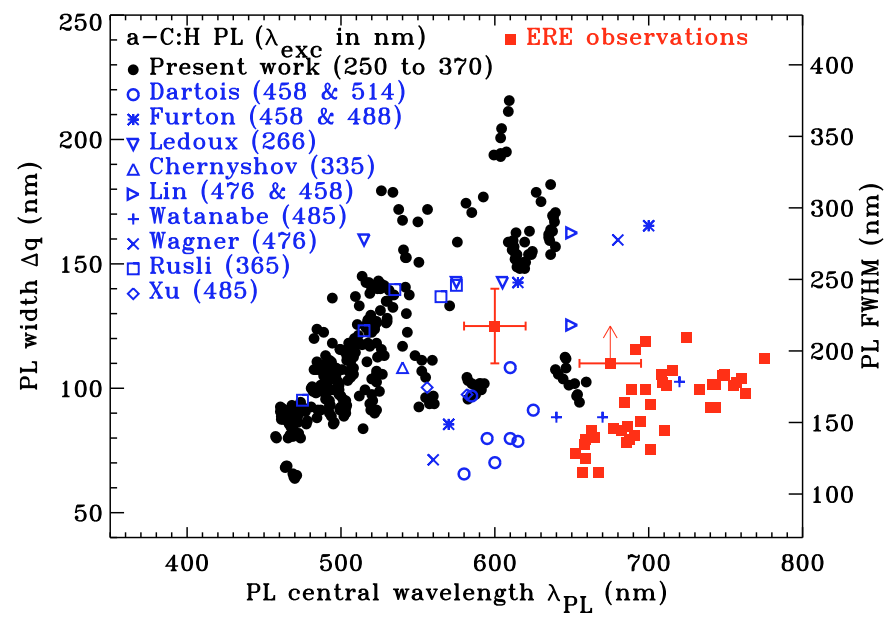

Fig. 13. Comparison between spectral characteristics (band width and central wavelength) of ERE (red filled squares) and a-C:H PL (black filled circles for our measurements and blue other symbols for a-C:H PL data coming from previous studies (Lin \& Feldman 1982; Lin \& Feldman 1983; Watanabe et al. 1982; Wagner \& Lautenschlager 1986; Chernyshov et al. 1991; Xu et al. 1993; Rusli et al. 1996; Ledoux et al. 2001; Dartois et al. 2005)). The ERE observations data come from Fig. 1 in Darbon et al. (1999), and we add the ERE observations corresponding to the DISM (Szomoru \& Guhathakurta 1998) (square with both $\Delta q$ and $\lambda$ error bars) and L780 dark nebula (Mattila 1979) (the arrow represents a $\Delta q$ lower limit).

nebula (Mattila 1979) for which the width and central wavelength determination are much less constrained.

It should be noted that the ERE spectral characteristics in DISM seem different from the other ERE detections: ERE in the DISM appears to occur at shorter wavelengths than in other environments and with a wider PL band. Szomoru \& Guhathakurta (1998) detected an ERE spectrum centered on $600 \mathrm{~nm}$, whereas $\lambda_{\mathrm{PL}}$ are between 650 and $700 \mathrm{~nm}$ in reflection nebulae (e.g. Witt \& Boroson 1990), around $700 \mathrm{~nm}$ in the dusty halo of M 82 (Perrin et al. 1995), between 700 and $780 \mathrm{~nm}$ in planetary nebulae (Furton \& Witt 1990, 1992) and HII regions (Perrin \& Sivan 1992; Sivan \& Perrin 1993; Darbon et al. 1998, 2000) and seems to be even above $800 \mathrm{~nm}$ in the Evil Eye galaxy (Pierini et al. 2002). Another ERE observation in high galactic latitude interstellar clouds made by Witt et al. (2008) (not plotted in Fig. 13 because no reliable width can be determined) seems to confirm that, in the DISM, the ERE emission peak wavelength lies around $600 \mathrm{~nm}$. As will be discussed in the next section, the DISM ERE observations are difficult and few, but if these detections are confirmed by future DISM observations, it shows the specific conditions and/or carriers of ERE in the diffuse interstellar medium environments.

In Fig. 13 we notice differences in PL properties with some of the a-C:H PL measurements from other studies, and this shows the wide range of a-C:H PL characteristics that can be obtained under different production conditions. Part of these differences in PL characteristics (within those with longer PL wavelength and narrower PL band) can be explained by the different wavelengths used to excite the PL (numbers appearing in parentheses in the figure). As we have seen (cf. Figs. 5 and 11), the PL central wavelength and width are constant when changing the excitation wavelength unless $\lambda_{\text {exc }}$ falls in the PL emission range. In this case, the PL band appears narrower and at longer wavelengths. Besides these PL spectral variations from one a-C:H sample to another, which can be explained by different excitation conditions, we point out that a rather wide variety of PL spectral characteristics exists for a-C:H, depending on the production conditions.

As previously found in laboratory (Watanabe et al. 1982; Furton \& Witt 1993), we also observed that the a-C:H PL band width is correlated to the PL central wavelength (Fig. 10) with a coefficient correlation of 0.86 . The same trend is observed for the ERE (Witt \& Boroson 1990 and Darbon et al. 1999 (Fig. 1) found a correlation coefficient of 0.52 and 0.68 , respectively). The "quartile defined" band widths $\Delta q$ we found for laboratory measurements are varying between 60 and $220 \mathrm{~nm}$ when choosing the width definition adopted by Witt \& Boroson (1990) and Darbon et al. (1999) for the ERE band, which is well adapted to astrophysical observations when the signal-to-noise ratio is low, but does not have any spectroscopic meaning. The adopted definition must be taken with care because the width value $\Delta q$ obtained by this method (difference between third and first quartiles of the band area, i.e., the width in which half of the total PL flux is concentrated) is largely different from the FWHM values: in the case of a hypothetical Gaussian emission band profile, the width defined with quartiles is 0.57 times the FWHM. Our a-C:H samples display PL band $F W H M$ between about 100 and $400 \mathrm{~nm}(\Delta q \in[50,200] \mathrm{nm}$, respectively).

The photoluminescence of a-C:H presents a continuous spectral variation that is one of the requirements for the ERE carriers but, except for the ERE observation in the DISM, the observed spectral PL characteristics for a-C:H and ERE do not totally agree. The widest optical gap $\left(E_{04}\right.$ greater than $\left.3.5 \mathrm{eV}\right)$ a-C:Hs do not correspond to the red ERE observations because their central wavelengths are too low. Some of our smaller gap a-C:Hs do not correspond either, because they seem to possess too large a PL band as compared to ERE spectra. In addition to the apparent agreement between ERE observations in the DISM and some a-C:H sample PL, it can be seen in Fig. 10 that a few of hydrogenated amorphous carbons PL overlap the ERE characteristics. In ERE observations, it is often difficult to determine the ERE baseline precisely (the instrument detection spectral range are most often not much larger than the ERE range), and it could increase the discrepancy with respect to laboratory measurements.

Our results show that varying the production conditions allows us to produce different a-C:H samples spanning a wide range of PL spectra (in Fig. 13, some samples exhibit the same $\Delta q$ of $100 \mathrm{~nm}$ for central emission from $480 \mathrm{~nm}$ to $660 \mathrm{~nm}$ ). It means that a wider range of PL spectral characteristics can be explored and accessed with different production conditions that we have not been able to create within our experimental system. In particular, a better control of the parameters might allow us to tailor a narrower PL band.

By taking optical effects of our sample geometry as a film into account, we obtained the intrinsic a-C:H PL spectra. We have to keep in mind that a-C:H dust PL must also undergo optical effects (see Sect. 5.5): self-absorption may also occur in the dust grain (the larger the dust grain, the more self-absorption will occur in ISM conditions (Mulas et al. 2004) and this will result in a narrowing and shift of the apparent PL spectrum toward longer wavelengths.

\subsection{Photoluminescence efficiencies}

The intrinsic PL absolute quantum yield of our a-C:H samples are lower than or equal to about $3 \%$ (the corresponding energetic 


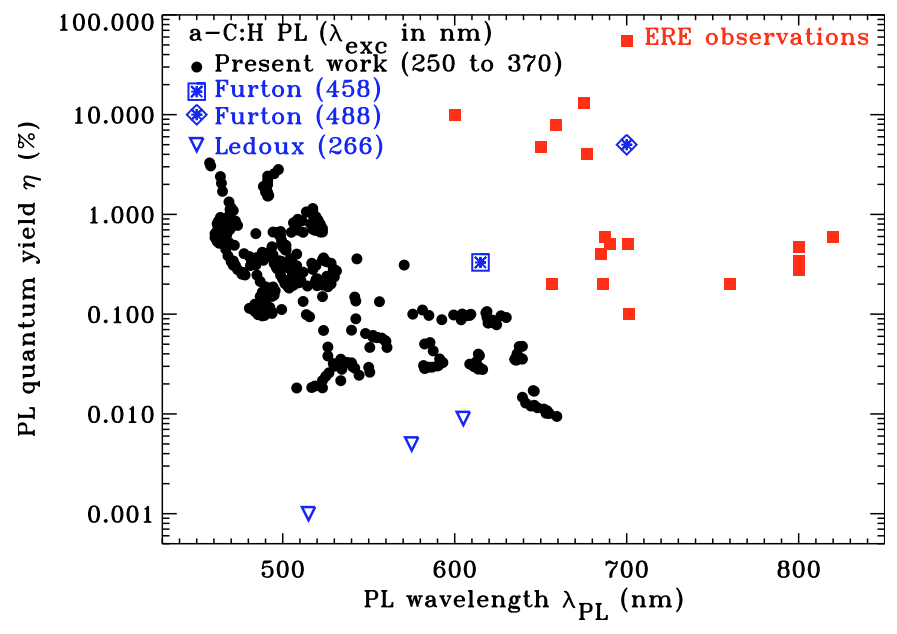

Fig. 14. Comparison between quantum yield $\eta$ and central wavelength $\lambda_{\mathrm{PL}}$ of ERE (red filled squares) and a-C:H PL (black filled circles for our measurements and blue other symbols for a-C:H PL data coming from laser excitation studies Furton \& Witt 1993; Furton et al. 1999; Ledoux et al. 2001). The ERE observations data come from Smith \& Witt (2002).

yields are lower than about $2 \%{ }^{4}$ ), and the a-C:H photoluminescence becomes less efficient when the optical gap decreases or when the PL band moves to longer wavelengths as shown in Figs. 7 and 9. It is important to note the importance to take the optical effects described in Sect. 3.1 into account because all of these effects, except the interferences effects, make the apparent yield ${ }^{5}$ lower than the intrinsic one. This increase in the internal yield as compared to the external one is significant (increase by a factor around 6) under our experimental measurement conditions.

Figure 7 shows that the higher a-C:H PL efficiencies correspond to the widest optical gap. However, we have seen in the previous section that widest gap a-C:H are those with the shortest PL wavelengths, hence those least corresponding to the ERE PL spectra. This difficulty of fulfilling ERE spectra and efficiencies together is shown in Fig. 14, which allows a comparison between a-C:H PL and ERE observations properties. The a-C:H PL measurements are those plotted in Fig. 9, and we also added the few absolute yield measurements from laser excitation studies (Furton \& Witt 1993; Furton et al. 1999; Ledoux et al. 2001). The ERE observations properties are reproduced from Table 1 and Fig. 4 in Smith \& Witt (2002). The yields we measured display, in a few case, large variations for the same PL color, well above uncertainties in the measurement. It probably means that higher quantum efficiencies can be obtained under specific conditions.

It should be noted that the a-C:H photoluminescence yield measurements from Ledoux et al. (2001) vary with the PL central wavelength in an opposite way to what is observed in a-C:H PL studies. The PL yields are up to three orders of magnitude lower than our measurements (when $\lambda_{\mathrm{PL}} \approx 510 \mathrm{~nm}$ ). This again shows the high PL quenching sensitivity of the a-C:H to their production and storage conditions. Furton \& Witt (1993) and Furton et al. (1999) also measured the photoluminescence from a-C:H and determined the corresponding absolute quantum

\footnotetext{
4 The variation between quantum and energetic yields is more important for PL occurring at longest wavelengths.

5 What we call the apparent or external yield is the one obtained when not taking the optical effects of the sample geometry and measurement configuration into account.
}

yields. Most of the previously discussed optical effects occurring in the a-C:H films are not accounted for. Although the a-C:H are supposed to be somewhat similar, efficiency variations over more than one order of magnitude are nevertheless observed between these two studies. The absolute efficiency from the Furton et al. (1999) measurement does not overlap other a-C:H measurements. This measurement does not agree either the correspondence between the PL efficiency and the optical gap (Fig. 7): the sample $E_{04}$ should be around $2.4 \mathrm{eV}$ since its Tauc gap is around $1.9 \mathrm{eV}$ (a shift of around $0.5 \mathrm{eV}$ is commonly observed between these two ways of defining the optical gap (e.g. Silva et al. 1996). The sample 5\% quantum yield is therefore three orders of magnitude more those we found for the corresponding optical gap. Furthermore, Furton \& Witt (1993), Furton et al. (1999), and Ledoux et al. (2001) (as well as the references used in Fig.13) used a laser as excitation source. One can wonder to what extent a laser excitation does not alter the samples, hence their PL spectrum. The samples PL efficiencies may be modified by this intense excitation. An a-C:H PL efficiency decrease was detected by Dartois et al. (2005), but the PL intensity was partly recovered when the laser exposure stopped for a few minutes. Within this regime, higher efficiencies are expected in an astrophysical context where photons are absorbed and relaxed one by one most of the time.

Most of the produced a-C:H exhibits photoluminescence with a quantum yield of the same order of magnitude as most of the ERE estimated yields, i.e., between $0.1 \%$ and $1 \%$. The same PL yield trend with the variation in the PL central wavelength is found for both $\mathrm{a}-\mathrm{C}: \mathrm{H}$ and ERE. The lowest $\mathrm{a}-\mathrm{C}: \mathrm{H}$ PL efficiencies correspond to the reddest PL band, and thus to a-C:H with the PL spectra that match the ERE observations better. The a-C:H we produced are not photoluminescent efficiently enough and red enough simultaneously. Furthermore, none of our a-C:H samples have a PL quantum yield higher than $10 \%$, as seems required for the ERE carriers by the DISM observations by Gordon et al. (1998) and Szomoru \& Guhathakurta (1998). The ERE quantum yield evaluation by Smith \& Witt (2002) also provided an even higher efficiency: the Red Rectangle nebula is treated apart but seems to have ERE efficiency of about $20 \%$ when employing the same method as for the other reflection nebulae.

In parallel, it is difficult to determine absolute PL efficiency from observations, because of the uncertain evaluation of light absorbed by the carriers estimated from the scattering. In addition, in some ISM environments (especially in the DISM), the extended red emission is very weak as compared to the background. Some underlying hypothesis are needed and little variations in these input parameters make the efficiency vary over more than an order of magnitude (e.g. Fig. 3 of Smith \& Witt 2002). The strong constraint for the ERE carrier candidates of a PL quantum yield greater than about $10 \%$ corresponds to the few and most difficult observations of the diffuse interstellar medium (DISM) ERE (Gordon et al. 1998; Szomoru \& Guhathakurta 1998). The first figure in Szomoru \& Guhathakurta (1998) shows this observational difficulty well: the night-sky emission spectrum displays an emission band in the spectral range of the detected ERE and its flux is more than two orders of magnitude greater than the ERE one. ERE efficiencies, which constitute a strong constraint for the ERE carriers, are determined under the assumption that the light scattered by dust (which allows one to evaluate the excitation absorbed) is isotropic, while the interstellar grains present a strongly forward-directed phase function. In the case of high galactic latitude interstellar clouds, where most of the diffuse interstellar medium observations occurred, 
illuminated by highly nonisotropic interstellar radiation field, this forward-scattering nature of interstellar grains sends most of the scattered light into directions other than the line of sight. Not taking this nonisotropic scattering into account, while the ERE is expected to be emitted isotropically, can give rise to an apparent reduction in the absorbed excitation by dust, and then to an apparent enhancement of the ERE yield in these regions, as outlined by Witt et al. (2008).

In addition to the photoluminescence efficiency discussed above, thermal emission involving the electronic levels associated with small carbon clusters may contribute to the overall apparent PL effect as discussed by Duley (2009). This blackbody radiation in the visible can only occur in the limit of small molecules or clusters since their internal temperature needs to be raised to thousands of degrees to play a significant role. Duley (2009) emphasizes that the thermal emission may dominate under far-UV photons excitation and that the combination of thermal and PL radiation would enhance the emission yield.

\subsection{Photoluminescence excitation}

We have seen (see Figs. 11 and 5) that the a-C:H photoluminescence spectrum, both the peak position and the band width, does not change with the explored excitation wavelength, expect when the excitation occurs at low enough energy to reach the PL emission band. In this case, the higher energy side of the PL band is not contributing to the spectrum anymore, but almost no change is observed in the reddest part of the spectrum. As a consequence, the PL band appears narrower, at longer wavelengths. This a-C:H PL effect has been previously observed (e.g. Chernyshov et al. 1991; Rusli et al. 1996; Pócsik \& Koós 2001).

Hydrogenated amorphous carbons have a PL quantum yield that does not vary with the excitation wavelength in the explored range, i.e., from $250 \mathrm{~nm}$ to the PL emission band wavelengths. Quantitative measurements was possible for excitation wavelengths up to $250 \mathrm{~nm}$ with our instruments, but we have also observed that a-C:H PL still exists for excitation wavelength down to $200 \mathrm{~nm}$. Excitation with a wavelength shorter than $200 \mathrm{~nm}$ was not possible with our set up, but it would be interesting to explore excitation in the vacuum UV spectral range. We estimate that most of the interstellar ERE-exciting photons correspond to our explored excitation wavelength range. We calculate the proportion of photons with wavelength in the explored 250-540 nm range as compared to the number of photons considered for the ERE excitation (Smith \& Witt 2002), i.e., with wavelength between $91 \mathrm{~nm}$ (ionization of $\mathrm{H}$ ) and $540 \mathrm{~nm}$ (edge of ERE band). This ratio is about $80-85 \%$ for the interstellar standard radiation field (i.e., the excitation source in the DISM, using the Mathis et al. (1983) prescription and more than $95 \%$ for the Red Rectangle spectral distribution, calculated from the source spectra of Sitko (1983), Reese \& Sitko (1996), and Schmidt et al. (1980). For a blackbody emission corresponding to a star later than $\mathrm{B} 8$ as the spectral energy distribution, this ratio is then more than about $80 \%$. The photoluminescence quantum yield of interstellar a-C:H in these environments would thus mainly be determined by the visible and near-UV exciting photons.

It has been suggested that ERE excitation occurs mainly for UV wavelengths lower than $250 \mathrm{~nm}$ (Witt \& Schild 1985; Darbon et al. 1999). Witt et al. (2006, 2009) have more recently suggested that the ERE results from a two-step process including its initiation, i.e., the creation/activation of the ERE carriers, and the ERE excitation. The ERE initiation, not the ERE excitation, needs the presence of energetic UV photons (Witt et al. 2006, 2009) because the energetic UV photon flux is too small to be the excitation source of the observed ERE, even with very high quantum efficiency. Instead, Witt et al. (2006, 2009) show that the ERE excitation must be due to the optical pumping of the ERE carriers by near UV/optical photons. We have shown that a-C:H photoluminesce when excited in this entire spectral range, with an approximatively constant efficiency.

This study shows that a-C:H photoluminescence is efficient on a very wide excitation band. That is probably an important requirement for the ERE carrier candidates since their PL efficiencies are determined considering all photons absorbed between the Lyman limit around $91 \mathrm{~nm}$ and the edge of ERE band around $540 \mathrm{~nm}$ as the ERE excitation (Smith \& Witt 2002). Therefore, a material that photoluminesces with high efficiency, but that only corresponds to a narrow range of excitation energies would hardly be able to match the ERE carrier requirements. This argument of a large and continuous absorption band from the visible PL band edge to $91 \mathrm{~nm}$ (13.6 eV in vacuum) supports solids or big molecules.

\subsection{Fatigue and oxygen PL quenching}

We observed that the PL efficiency of some of our a-C:H samples is time dependent when exposed to UV light in an oxygen atmosphere. A more or less strong decrease in the PL intensity is seen when the sample is exposed to UV/visible excitation for several minutes. In some cases, we clearly observed this PL diminution with the shape of the excitation area on the sample, when placing it under the UV lamp. Ideally, it would be better to measure the $\mathrm{PL}$ of a-C:H in situ, i.e., without putting the samples in air after their production. Being aware of this fatiguing effect, we take care to measure the PL spectra before any other sample measurement, and just after the film is made.

We have not determined whether this fatiguing effect of the a-C:H photoluminescence is activated or accelerated by the photons irradiation and whether this effect only occurs when the sample is in air, especially when exposed to oxygen. It is possible that oxygen reacts with the a-C:H by photochemistry induced by the excitation source. Sakata et al. (1992) noted also a fatiguing effect for a related material (QCC). According to their observations, it seems that UV irradiation only accelerates the fatigue that also occurs (but in days instead of hours) when the QCC sample is in air without photons. They do not detect any significant fatigue when the sample is conserved in dark and in vacuum.

Xu et al. (1993) measured the a-C:H PL fatigue under visible $(2.54 \mathrm{eV})$ photon irradiation. They showed that this effect is more or less strong (20\% to more than a $90 \%$ reduction in the PL intensity) from one sample to the next. It appears that the widest optical gap samples (i.e., the ones with the highest PL yields) are those that are more affected by the fatiguing effect textbf(we also observed this effect). Xu et al. (1993) also observed that the fatiguing is reduced when the PL measurement occurs at lower temperatures.

Laikhtman et al. (1998) studied the effect of hydrogenation and oxidation on the absolute PL yield of diamond films. The PL efficiency values are found to depend on the state of the surface: hydrogenated terminated films exhibit the highest quantum yields, whereas oxidation results in degradation of the photoluminescence efficiencies. Even a small amount of oxygen (around one atomic percent) strongly lowers the PL yield by a factor two to three.

An issue arises because the experiments are not performed under ultra-high vacuum conditions such as in space. In particular, we detect trace amount of oxygen via a shallow carbonyl 
IR absorption. The presence of a low amount of oxygen in the $\mathrm{a}-\mathrm{C}: \mathrm{H}$ network, either due to the production or the post production exposition to air, can severely degrade the quantum yield by scavenging the electron produced by the excitation, creating a trap.

\subsection{Comparison of photoluminescence by a-C:H film produced in laboratory and photoluminescence of a-C:H in interstellar conditions}

In our experiments, the a-C:H samples we obtained are films of a few micrometers thickness deposited on a substrate, and the PL measurements are done in air at room temperature. But $\mathrm{a}-\mathrm{C}: \mathrm{H}$ dust in interstellar medium exists under different conditions (shape, size, temperature, pressure, radiation field, etc.) from those of our laboratory experiments. Therefore, we have to wonder whether these differences have any effect on the a-C:H photoluminescence and to what extent we can compare laboratory PL measurement with a-C:H PL in interstellar conditions. With the optical effects described in Sect. 3.1 and in Appendix B taken into account in our PL measurements analysis, we obtained the intrinsic a-C:H PL properties (spectra and efficiencies), thereby avoiding effects of the sample geometry and the experiment configuration.

Malloci et al. (2004) and Mulas et al. (2004) took this issue into consideration. They model effects of modification between PL laboratory experiments performed for bulk samples and PL produced by a size distribution of homogeneous, spherical dust grain. Like us, they modeled photoluminescence by emission from incoherent, unpolarized density of oscillating electric dipoles in the local case approximation, i.e., considering that excitation energy is locally absorbed and that the resulting PL is emitted at the same location as the absorption. This seems to be a good approximation in the case of a-C:H since the PL mechanism appears to be produced by confined clusters of $\mathrm{sp}^{2}$ sites in the $\mathrm{sp}^{3}$ matrix (Demichelis et al. 1995; Robertson 1996). They applied their model to HAC particles and discussed the astrophysical implications about extended red emission. They found that geometrical and size effects affect PL resulting from small interstellar particles. PL spectra are modulated by self-absorption at shorter wavelengths, occurring when particles are not too small. Then, different size distributions give rise, or not, to a redshift of the PL band depending on the presence (or not) of smaller particles in the size distribution. Finally, they found that the influence of the size and geometrical effects on dust PL exists and depends on the particles size distribution and on the angle between the directions of excitation and emission. This implies different consequences on the ERE phenomenon. First, a variation in the size distribution of the same material between different locations (within a same interstellar object or in different interstellar environments) could give rise to different apparent PL properties as observed for ERE. Second, ERE could appear redder than the intrinsic photoluminescence of its carrier. This apparent reddening could be more or less strong depending on the size distribution of the ERE carriers. For a fixed grain size, the self absorption diminishes when the optical gap increases because the shift between absorption and emission band becomes greater at wider optical band gap. As a consequence, the redder is the a-C:H PL, the even redder will be the PL from an a-C:H dust grain in which self absorption occurs.

The PL measurements were carried out at room temperature. One can wonder whether the difference between ISM temperature and room temperature have any impact on the PL of interstellar a-C:H dust. Watanabe et al. (1982),
Lin \& Feldman (1983), Chernyshov et al. (1991), and Xu et al. (1993) have studied the temperature's influence on the a-C:H PL and conclude that the PL intensity increases when the sample temperature decreases, but this increase is moderate compared to other materials such as a-Si:H for which Chernyshov et al. (1991) observed an PL intensity increase of more than four magnitude orders between room temperature and $50 \mathrm{~K}$. Nevertheless, an intensity increases by a factor lower than or equal to two is observed when temperature falls from $350 \mathrm{~K}$ to $2 \mathrm{~K}$, except for one of the of $\mathrm{Xu}$ et al. samples for which the PL efficiency increases by a factor of ten when cooling the sample from $300 \mathrm{~K}$ to $120 \mathrm{~K}$. $\mathrm{Xu}$ et al. (1993) show that this enhancement of PL efficiency does not affect all a-C:H samples in the same way. This efficiency gain is concomitant to a reduction of the fatiguing effect at lower temperatures. The a-C:H PL quantum yield should thus increase at lower temperatures but probably not so much because the PL temperature dependence of a-C:H is weak. Chernyshov et al. (1991) also observed a small narrowing of the PL band for low temperatures, but no change in the PL spectrum shape is observed by others.

\subsection{Review of the comparison between a-C:H photoluminescence and ERE observations}

In this last section of the discussion, we would like to review the different arguments for and against the hydrogenated amorphous carbons as the extended red emission carriers. The hydrogenated amorphous carbons are already known to be an abundant interstellar material. Spectral features associated with this component are widely observed in the DISM $(3.4,6.85$, and 7.25 microns absorption bands). The a-C:H photoluminescence spans a wide range of spectral variation (PL band position, width, and intensity) in response to varying the production/treatment conditions. This is in good agreement with the ERE spectral variations from one interstellar object to another and within a given object caused by the different environment conditions (density and hardness of the radiation field, temperature, etc.). Moreover, a-C:H exhibit luminescence bands whose width increases with the central wavelength as observed for the extended red emission. This is not the only correlation that matches the ERE observations: for both a-C:H photoluminescence and ERE observations, the respectively measured and estimated efficiencies decrease when the PL band occurs at longer wavelengths. The high ERE efficiencies, estimated considering the ERE carriers responsible for all the absorption between 91 and $550 \mathrm{~nm}$, require that the ERE producing material has a wide absorption band giving rise to PL. For a-C:H, we showed that at least the near UV and visible part of the excitation spectrum gives rise to efficient PL: the PL efficiency does not decrease for excitation energy up to $5 \mathrm{eV}$. The higher energy part still needs to be explored, but the near UV and optical spectral range seems to be the one responsible for the ERE excitation.

However, PL spectral characteristics and efficiency of a-C:H does not seem to fully agree with the ERE properties estimated from astrophysical observations. The PL spectrum of our a-C:H samples is either slightly too large or occurs at wavelengths that are slightly too short to correspond exactly to the ERE observations. The quantum yields that we deduce are compatible with most of quantum yield limits set by ERE observations, but they are not compatible with the highest quantum yield of $10 \%$ set by the few observations of the weakly emitting DISM. Moreover, the a-C:H higher quantum efficiencies correspond to the less red PL bands. Therefore, there is a difficulty to simultaneously meet the ERE spectra and the ERE high efficiencies. 
Nevertheless, effects such as the PL fatigue, the oxygen content during production or post production oxidation quenching the quantum yield, the influence of PL measurement temperature, and the influence of the dust grains geometry and size seem to more or less affect the apparent photoluminescence properties. Furthermore, all of these effects seem to modify the PL characteristics in such a way that a-C:H emission will approach the ERE properties. Thus, they still need to be investigated further to obtain a complete and definitive answer to the question: could the interstellar a-C:H participate in the extended red emission?

\section{Conclusions}

We performed the first measurements of absolute PL quantum yield as a function of the excitation wavelength for hydrogenated amorphous carbons laboratory analogs to interstellar dust. To obtain intrinsic internal and absolute PL efficiencies, we took the optical effects caused by the sample's geometry into account. A link between the variations in PL properties (efficiency, central wavelength, and band width) and the optical gap energy, which characterizes the produced a-C:H materials, was established quantitatively. These properties were compared to the interstellar observations of the extended red emission.

The a-C:H photoluminescence spans a wide range of spectral variation, wider than the one explored with our experimental set up. The PL band width increases with its central wavelength (Fig. 13). The same trend is observed for ERE. Some a-C:H PL measurements and ERE observations match, but redder a-C:H PL would be better for a complete PL spectral characteristics overlap.

The absolute yield increases with the gap (Fig. 7), hence with the PL emission central wavelength. Such a trend is also observed for ERE detections (Fig. 14, see also the combination of Figs. 2 and 4 in Smith \& Witt 2002). There would be agreement if the observed yields were one or two orders of magnitude lower.

The PL spectrum (central wavelength and width) and efficiency remain approximatively constant when excited in the near UV/optical spectral range (Figs. 11 and 12). This wide absorption band for a-C:H is a favorable characteristic to give rise to high PL efficiency for the ERE. The near UV and visible photons seems to be those responsible for the carriers optical pumping giving rise to ERE.

Until now and ever since the ERE first detection in 1975, this observed feature remains an unsolved problem in astrophysics. No satisfactory material completely fulfills the observational requirements for the extended red emission carrier. Hydrogenated amorphous carbons also seem have some difficulty simultaneously satisfying the high quantum yield criteria and PL spectral characteristics. However, it seems that several complex effects, such as the shape and size of interstellar dust grains, for example, could shift the PL properties that we observed in the right direction to correspond to the ERE observations.

The strongest astrophysical constraint set by observations concerns the evaluation of ERE efficiencies. Large uncertainties still exist in the evaluation of photons truly absorbed by the ERE carriers, relying today on grain scattering assumed properties. For the laboratory point of view, some points should be addressed in more detail in the future concerning the PL quantum-yield behavior at low temperatures and the effect of small amount of oxygen atoms in the a-C:H network on the PL quenching.

Acknowledgements. We would like to thank Dominique Deboffle for his daily help on experimental developments, Vincent Guerrini for his patience in making dedicated vacuum vessels, Noel Coron for his scientific enthusiasm and numerous loans of equipment. We would also like to thank Louis d'Hendecourt, Thomas Pino, Philippe Brchignac, Yvain Carpentier, and Graldine Fraud for fruitful exchanges (both for experiments and discussions!). Finally, we acknowledge W. Duley for helpful comments on this paper.

\section{References}

Berné, O., Joblin, C., Rapacioli, M., et al. 2008, A\&A, 479, L41

Bourée, J. E., Heitz, T., Godet, C., et al. 1998, Journal of Non-Crystalline Solids, 227,574

Bubenzer, A., Dischler, B., Brandt, G., \& Koidl, P. 1983, J. Appl. Phys., 54, 4590

Chang, H.-C., Chen, K., \& Kwok, S. 2006, ApJ, 639, L63

Chernyshov, S., Terukov, E., Vassilyev, V., \& Volkov, A. 1991, Journal of NonCrystalline Solids, 134, 218

Chlewicki, G., \& Laureijs, R. J. 1987, in NATO ASIC Proc. 191: Polycyclic Aromatic Hydrocarbons and Astrophysics, ed. A. Leger, L. D'Hendecourt, \& N. Boccara, 335

Choi, S. 2001, Appl. Surf. Sci., 169, 217

Cohen, M., Anderson, C. M., Cowley, A., et al. 1975, ApJ, 196, 179

Darbon, S., Perrin, J., \& Sivan, J. 1998, A\&A, 333, 264

Darbon, S., Perrin, J.-M., \& Sivan, J.-P. 1999, A\&A, 348, 990

Darbon, S., Zavagno, A., Perrin, J., et al. 2000, A\&A, 364, 723

Dartois, E., Muñoz Caro, G. M., Deboffle, D., \& D’Hendecourt, L. 2004, A\&A, 423, L33

Dartois, E., Muñoz Caro, G. M., Deboffle, D., Montagnac, G., \& D’Hendecourt, L. 2005, A\&A, 432, 895

De Mello, J. C., Wittmann, H. F., \& Friend, R. H. 1997, Advanced Materials, 9, 230

Demichelis, F., Schreiter, S., \& Tagliaferro, A. 1995, Phys. Rev. B, 51, 2143

D’Hendecourt, L., Leger, A., Olofsson, G., \& Schmidt, W. 1986, A\&A, 170, 91

Dischler, B., Bubenzer, A., \& Koidl, P. 1983, Appl. Phys. Lett., 42, 636

Duley, W. W. 1985, MNRAS, 215, 259

Duley, W. W. 1988, Ap\&SS, 150, 387

Duley, W. W. 2009, ApJ, 705, 446

Duley, W. W., \& Williams, D. A. 1988, MNRAS, 230, 1P

Duley, W. W., Seahra, S., \& Williams, D. A. 1997, ApJ, 482, 866

Duley, W. W., Scott, A. D., Seahra, S., \& Dadswell, G. 1998, ApJ, 503, L183

Furton, D. G., \& Witt, A. N. 1990, ApJ, 364, L45

Furton, D. G., \& Witt, A. N. 1992, ApJ, 386, 587

Furton, D. G., \& Witt, A. N. 1993, ApJ, 415, L51

Furton, D. G., Laiho, J. W., \& Witt, A. N. 1999, ApJ, 526, 752

Gordon, K. D., Witt, A. N., \& Friedmann, B. C. 1998, ApJ, 498, 522

Heitz, T., Godet, C., Bourée, J. E., Drévillon, B., \& Conde, J. P. 1999, Phys. Rev. B, 60, 6045

Holm, R. T., McKnight, S. W., Palik, E. D., \& Lukosz, W. 1982, Appl. Opt., 21, 2512

Jarman, R. H., Ray, G. J., Standley, R. W., \& Zajac, G. W. 1986, Appl. Phys. Lett., 49, 1065

Kassavetis, S., Patsalas, P., Logothetidis, S., Robertson, J., \& Kennou, S. 2007, Diamond and Related Materials, 16, 1813

Kleber, R. 1991, Thin Solid Films, 205, 274

Kovalenko, S. 2001, Semiconductor Physics, Quantum Electronics and Optoelectronics, 4, 214

Laikhtman, A., Hoffman, A., Kalish, R., et al. 1998, Appl. Phys. Lett., 73, 1433 Lazar, G. 1998, Acta Physica Polonica A, 100, 67

Ledoux, G., Ehbrecht, M., Guillois, O., et al. 1998, A\&A, 333, L39

Ledoux, G., Guillois, O., Huisken, F., et al. 2001, A\&A, 377, 707

Lin, S.-H., \& Feldman, B. J. 1982, Phys. Rev. Lett., 48, 829

Lin, S.-H., \& Feldman, B. J. 1983, Philosophical Magazine Part B, 47, 113

Lukosz, W. 1981, J. Opt. Soc. Am. (1917-1983), 71, 744

Malloci, G., Mulas, G., \& Benvenuti, P. 2004, A\&A, 420, 809

Mathis, J. S., Mezger, P. G., \& Panagia, N. 1983, A\&A, 128, 212

Mattila, K. 1979, A\&A, 78, 253

Mulas, G., Malloci, G., \& Benvenuti, P. 2004, A\&A, 420, 921

Nollau, A., Hoffmann, M., Floreck, K., Fritz, T., \& Leo, K. 2000, J. Appl. Phys., 87, 7802

Pendleton, Y. J., \& Allamandola, L. J. 2002, ApJS, 138, 75

Perrin, J., \& Sivan, J. 1992, A\&A, 255, 271

Perrin, J.-M., Darbon, S., \& Sivan, J.-P. 1995, A\&A, 304, L21

Pierini, D., Majeed, A., Boroson, T. A., \& Witt, A. N. 2002, ApJ, 569, 184

Pócsik, I., \& Koós, M. 2001, Diamond and Related Materials, 10, 161

Reese, M. D., \& Sitko, M. L. 1996, ApJ, 467, L105

Rhee, Y. M., Lee, T. J., Gudipati, M. S., Allamandola, L. J., \& Head-Gordon, M. 2007, Proceedings of the National Academy of Sciences, 104, 5274

Ristein, J., Stief, R. T., Ley, L., \& Beyer, W. 1998, J. Appl. Phys., 84, 3836

Robertson, J. 1986, Adv. Phys., 35, 317 
Robertson, J. 1996, Phys. Rev. B, 53, 16302

Rodnyi, P. 1997, Physical processes in inorganic scintillators (CRC Press)

Rusli, R., Robertson, J., \& Amaratunga, G. A. J. 1996, J. Appl. Phys., 80, 2998

Sakata, A., Wada, S., Narisawa, T., et al. 1992, ApJ, 393, L83

Sandford, S. A., Allamandola, L. J., Tielens, A. G. G. M., et al. 1991, ApJ, 371, 607

Schmidt, G. D., Cohen, M., \& Margon, B. 1980, ApJ, 239, L133

Schütte, S., Will, S., Mell, H., \& Fuhs, W. 1993, Diamond and Related Materials, 2, 1360

Schwarz-Selinger, T., von Keudell, A., \& Jacob, W. 1999, J. Appl. Phys., 86, 3988

Seahra, S. S., \& Duley, W. W. 1999, ApJ, 520, 719

Seth, J., \& Babu, S. V. 1993, J. Appl. Phys., 73, 2496

Silva, S. R. P., Robertson, J., Rusli, G., et al. 1996, Philosophical Magazine Part B, 74, 369

Sitko, M. L. 1983, ApJ, 265, 848

Sivan, J., \& Perrin, J. 1993, ApJ, 404, 258

Smith, T. L., \& Witt, A. N. 2002, ApJ, 565, 304

Spoon, H. W. W., Armus, L., Cami, J., et al. 2004, ApJS, 154, 184

Swanepoel, R. 1983, J. Phys. E: Scientific Instruments, 16, 1214

Szomoru, A., \& Guhathakurta, P. 1998, ApJ, 494, L93

Tamor, M. A., \& Vassell, W. C. 1994, J. Appl. Phys., 76, 3823
Turlo, J., \& Rozwadowskajasniewska, K. 1987, Journal of Non Crystalline Solids, 90, 641

Vijh, U. P., Witt, A. N., \& Gordon, K. D. 2005, ApJ, 633, 262

Wada, S., Mizutani, Y., Narisawa, T., \& Tokunaga, A. T. 2009, ApJ, 690, 111

Wagner, J., \& Lautenschlager, P. 1986, J. Appl. Phys., 59, 2044

Watanabe, I., Hasegawa, S., \& Kurata, Y. 1982, Japan. J. Appl. Phys., 21, 856

Weber, M. J., \& Monchamp, R. R. 1973, J. Appl. Phys., 44, 5495

Webster, A. 1993, MNRAS, 264, L1+

Witt, A. N., \& Schild, R. E. 1985, ApJ, 294, 225

Witt, A. N., \& Schild, R. E. 1988, ApJ, 325, 837

Witt, A. N., \& Boroson, T. A. 1990, ApJ, 355, 182

Witt, A. N., \& Vijh, U. P. 2004, in Astrophysics of Dust, ed. A. N. Witt, G. C. Clayton, \& B. T. Draine, ASP Conf. Ser., 309, 115

Witt, A. N., Gordon, K. D., \& Furton, D. G. 1998, ApJ, 501, L111

Witt, A. N., Gordon, K. D., Vijh, U. P., et al. 2006, ApJ, 636, 303

Witt, A. N., Mandel, S., Sell, P. H., Dixon, T., \& Vijh, U. P. 2008, ApJ, 679, 497

Witt, A. N., Vijh, U. P., Hobbs, L. M., et al. 2009, ApJ, 693, 1946

Xu, S., Hundhausen, M., Ristein, J., Yan, B., \& Ley, L. 1993, proceedings of the Fifteenth International Conference on Amorphous Semiconductors-Science and Technology, Journal of Non-Crystalline Solids, 164, 1127

Yoshimi, M., Shimizu, H., Hattori, K., Okamoto, H., \& Hamakawa, Y. 1992, Optoelectronics, 7, 69

Pages 15 to 24 are available in the electronic edition of the journal at http://www . aanda.org 

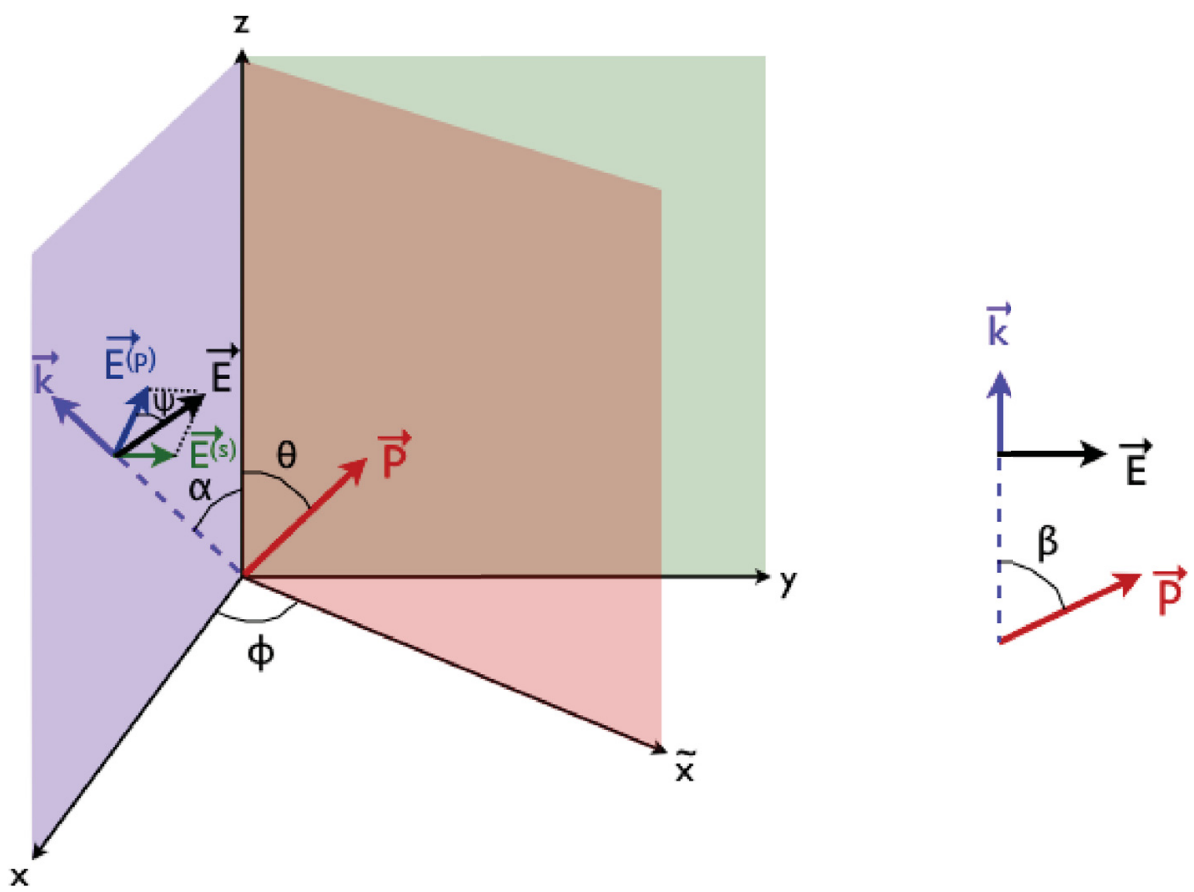

Table A.1. Definition of axis, angles, and other notations.

\begin{aligned} \hline \hline Notation & Definition \\ \hline 0 & Subscript applying to the film medium. \\ 1 & Subscript applying to the medium above the film (air). \\ 2 & Subscript applying to the medium below the film (substrat). \\ $(\mathrm{s}) & \begin{array}{l}\text { Superscript applying to the direction of polarization of the electric field } \\ \text { perpendicular to the observation plane }\left(x, z, \boldsymbol{k}_{j}\right)\end{array} \\ (\mathrm{p}) & \begin{array}{l}\text { Superscript applying to the direction of polarization of the electric field } \\ \text { in the observation plane }\left(x, z, \boldsymbol{k}_{j}\right) \text { and perpendicular to } \boldsymbol{k}_{j}\end{array} \\ z$-axis & Direction perpendicular to the surface of the film. \\ $x$-axis & Direction both in the film surface and in the plane in which the wave vector $\boldsymbol{k}$ is. \\ $\hat{x}$-axis & Direction both in the film surface and in the plane in which the dipole moment $\boldsymbol{p}$ is. \\ $\alpha_{j} &$ Observation angle in medium $j$, between $\boldsymbol{k}_{j}$ and the $z$-axis. \\ $\theta &$ Angle between $\boldsymbol{p}$ and the $z$-axis. \\ $\phi &$ Angle between the $\hat{x}$-axis and the $x$-axis. \\ $\beta &$ Angle between $\boldsymbol{p}$ and $\boldsymbol{k}_{\mathbf{0}} \\ \psi &$ Angle between $\boldsymbol{E}_{\mathbf{0}}$ and $\boldsymbol{E}_{\mathbf{0}}^{(\mathbf{p})} \\ \tilde{n}_{j} &$ Complex refractive index of the medium $j \\ . n_{j} &$ Real part of the refractive index of the medium $j \\ . \boldsymbol{\kappa}_{j} &$ Imaginary part of the refractive indice of the medium $j \\ . \tilde{k}_{j} &$ Complex wave vector in medium $j \\ . \boldsymbol{E} &$ Electric field created by an electric dipole in an infinite medium. \end{aligned}

\section{Appendix A: Emission by an electric dipole in an infinite medium}

We first study the electric field $\boldsymbol{E}$ created by a single electric dipole, with dipole moment $\boldsymbol{p}$, in an infinite homogenous medium, and its decomposition into s- and p-polarized components. The configuration is described by Fig. A.1 and many notations used are listed in Table A.1.

The refractive index $\tilde{n}_{j}$ is complex if the medium $j$ is absorbing. In the case of considering a non-absorbing medium $j$, the imaginary part of the index, $\kappa_{j}$, is zero. The refractive index $\tilde{n}_{j}$ is given by the relation A.1, and the wave vector $\tilde{k}_{j}$ (in the direction of the observator) is linked to the complex refractive index by Eq. (A.2):

$\tilde{n}_{j}=n_{j}+\mathrm{i} \kappa_{j}$

$\tilde{k}_{j}=\tilde{n}_{j} \cdot \frac{2 \pi}{\lambda} \quad$ where $\lambda$ is the wave length in vacuum. 


\section{A.1. Polarization of the electric field}

The electric field $\boldsymbol{E}$ is perpendicular to the direction of observation $\boldsymbol{k}$ and can be decomposed into two orthogonal components, i.e., two polarizations: the s-polarized one is perpendicular to the observation plane and the (p)-polarized one is in this plane:

$\boldsymbol{E}^{(\mathrm{s})}=E^{(\mathrm{s})} \boldsymbol{u}_{\boldsymbol{y}}$

$\boldsymbol{E}^{(\mathrm{p})}=E^{(\mathrm{p})}\left(-\cos \alpha \boldsymbol{u}_{\boldsymbol{x}}+\sin \alpha \boldsymbol{u}_{z}\right)$

$E^{(\mathrm{s})}=E \sin \psi \quad$ and $\quad E^{(\mathrm{p})}=E \cos \psi$

$\boldsymbol{E}=E \mid \begin{array}{lr}-\cos \psi \cdot \cos \alpha \boldsymbol{u}_{\boldsymbol{x}} \\ \sin \psi & \boldsymbol{u}_{\boldsymbol{y}} \\ \cos \psi \cdot \sin \alpha & \boldsymbol{u}_{z}\end{array}$

$E^{(\mathrm{s})}=E_{y} \quad$ and $\quad E^{(\mathrm{p})}=E_{z} \sin \alpha-E_{x} \cos \alpha$

$\boldsymbol{u}_{\boldsymbol{p}}=\sin \beta \boldsymbol{u}_{\boldsymbol{E}}+\cos \beta \boldsymbol{u}_{\boldsymbol{k}}$

$\boldsymbol{E}=E\left[\frac{1}{\sin \beta} \boldsymbol{u}_{\boldsymbol{p}}-\frac{\cos \beta}{\sin \beta} \boldsymbol{u}_{\boldsymbol{k}}\right]$

$\boldsymbol{E}=\frac{E}{\sin \beta}\left(\begin{array}{l|l}\sin \theta \cdot \cos \phi \boldsymbol{u}_{\boldsymbol{x}} \\ \sin \theta \cdot \sin \phi \boldsymbol{u}_{\boldsymbol{y}} \\ \cos \theta \cos \beta & \boldsymbol{u}_{z}\end{array} \mid \begin{array}{l}\sin \alpha \boldsymbol{u}_{\boldsymbol{x}} \\ 0 \\ \cos \alpha \boldsymbol{u}_{\boldsymbol{z}}\end{array}\right)$

$\boldsymbol{E}=\frac{E}{\sin \beta} \mid \begin{array}{lr}\sin \theta \cdot \cos \phi-\sin \alpha \cdot \cos \beta \boldsymbol{u}_{\boldsymbol{x}} \\ \sin \theta \cdot \sin \phi & \boldsymbol{u}_{\boldsymbol{y}} \\ \cos \theta-\cos \alpha \cdot \cos \beta & \boldsymbol{u}_{z} .\end{array}$

With Eqs. (A.7) and (A.8), we find

$E^{(\mathrm{s})}=\frac{E}{\sin \beta} \sin \theta \sin \phi$
$E^{(\mathrm{p})}=\frac{E}{\sin \beta}(\cos \theta \sin \alpha-\sin \theta \cos \phi \cos \alpha)$.

\section{A.2. Radiant intensity in an infinite medium}

The next equations show how to obtain the intensity from the electric field amplitude. We can linked the electric field to the irradiance that is the incident power of electromagnetic radiation at a surface, per unit area.

Specific $^{6}$ irradiance $\left(\mathrm{W} \cdot \mathrm{m}^{-2} \cdot \mathrm{nm}^{-1}\right): \quad \operatorname{Irr}=\langle\boldsymbol{\Pi}\rangle=\frac{1}{2} \operatorname{Re}\left(\boldsymbol{E} \times \boldsymbol{H}^{*}\right)$

$$
\operatorname{Irr}=\frac{n \epsilon_{0} c}{2}\|E\|^{2}
$$

where $\epsilon_{0}$ is the vacuum permittivity, $c$ the speed of light in vacuum, and $n$ the refractive index of the medium.

Radiant intensity (incident power per unit solid angle) $I$ and irradiance created by the electric dipole $\boldsymbol{p}$ are normalized as

$$
\begin{aligned}
\iint_{4 \pi}\left(I_{p}^{(\mathrm{s})}+I_{p}^{(\mathrm{p})}\right) \cdot \mathrm{d} \Omega & =\iint_{4 \pi r^{2}}\left(\operatorname{Irr}_{p}^{(\mathrm{s})}+\operatorname{Irr}_{p}^{(\mathrm{p})}\right) \cdot \mathrm{d} S=P(\lambda) \\
I_{p}^{(\mathrm{s}, \mathrm{p})} \cdot \mathrm{d} \Omega & =\operatorname{Irr}_{p}^{(\mathrm{s}, \mathrm{p})} \cdot \mathrm{d} S=\operatorname{Irr}_{p}^{(\mathrm{s}, \mathrm{p})} \cdot r^{2} \mathrm{~d} \Omega
\end{aligned}
$$

where $r$ is the radius of the sphere where the irradiance is calculated, and $P(\lambda)$ is the total ${ }^{7}$ power emitted at the wavelength $\lambda$ by a single dipole $\boldsymbol{p}$, in all directions of the space.

Specific radiant intensity $\left(\mathrm{W} \cdot \mathrm{sr}^{-1} \cdot \mathrm{nm}^{-1}\right): I_{p}^{(\mathrm{s}, \mathrm{p})}=\operatorname{Irr}_{p}^{(\mathrm{s}, \mathrm{p})} \cdot r^{2}=r^{2} \cdot \frac{n \epsilon_{0} c}{2}\left\|E^{(\mathrm{s}, \mathrm{p})}\right\|^{2}$

$$
I_{p}^{(\mathrm{s}, \mathrm{p})}=F \cdot \frac{\sin ^{2} \beta}{E^{2}} \cdot\left\|E^{(\mathrm{s}, \mathrm{p})}\right\|^{2}
$$

$I_{p}^{(\mathrm{s})}=F \sin ^{2} \theta \sin ^{2} \phi \quad$ and $I_{p}^{(\mathrm{p})}=F(\cos \theta \sin \alpha-\sin \theta \cos \phi \cos \alpha)^{2}$.

\footnotetext{
${ }^{6}$ The adjective specific means that the variable is in unit of wavelength.

7 The contribution of polarizations (s) and (p) summed.
} 
The normalization Eq. (A.11) let us obtain factor $F$ :

$F \int_{0}^{\pi} \int_{0}^{2 \pi}\left(\sin ^{2} \theta \sin ^{2} \phi+(\cos \theta \sin \alpha-\sin \theta \cos \phi \cos \alpha)^{2}\right) \cdot \sin \alpha \mathrm{d} \alpha \mathrm{d} \phi=P(\lambda)$

$$
\begin{aligned}
\frac{P(\lambda)}{F}= & \sin ^{2} \theta \int_{0}^{\pi} \sin \alpha \mathrm{d} \alpha \int_{0}^{2 \pi} \sin ^{2} \phi \mathrm{d} \phi+\cos ^{2} \theta \int_{0}^{\pi} \sin ^{3} \alpha \mathrm{d} \alpha \int_{0}^{2 \pi} \mathrm{d} \phi \\
& +\sin ^{2} \theta \int_{0}^{\pi} \cos ^{2} \alpha \sin \alpha \mathrm{d} \alpha \int_{0}^{2 \pi} \cos ^{2} \phi \mathrm{d} \phi-2 \cos \theta \sin \theta \int_{0}^{\pi} \sin ^{2} \alpha \cos \alpha \mathrm{d} \alpha \int_{0}^{2 \pi} \cos \phi \mathrm{d} \phi \\
\frac{P(\lambda)}{F}= & \sin ^{2} \theta[-\cos \alpha]_{0}^{\pi} \cdot\left[\frac{\phi}{2}-\frac{\sin 2 \phi}{4}\right]_{0}^{2 \pi}+2 \pi \cos ^{2} \theta\left[-\cos \alpha+\frac{\cos ^{3} \alpha}{3}\right]_{0}^{\pi} \\
& +\sin ^{2} \theta\left[-\frac{\cos ^{3} \alpha}{3}\right]_{0}^{\pi} \cdot\left[\frac{\phi}{2}+\frac{\sin 2 \phi}{4}\right]_{0}^{2 \pi}-2 \cos \theta \sin \theta\left[-\frac{\sin ^{3} \alpha}{3}\right]_{0}^{\pi} \cdot[\sin \phi]_{0}^{2 \pi} \\
\frac{P(\lambda)}{F}= & 2 \pi \sin ^{2} \theta+\frac{8 \pi}{3} \cos ^{2} \theta+\frac{2 \pi}{3} \sin ^{2} \theta+0 \\
F=\frac{3}{8 \pi} & P(\lambda)=r^{2} \cdot \frac{n \epsilon_{0} c}{2} \frac{E^{2}}{\sin ^{2} \beta} .
\end{aligned}
$$

The radiant intensity emitted by one electric dipole $\boldsymbol{p}$, in the direction $\boldsymbol{k}$ given by the angles $(\alpha, \phi)$, is given by the next equation (whatever the distance from source dipole since the medium is infinite, homogenous and not absorbing):

$I_{p}^{(\mathrm{s})}(\lambda)=\frac{3}{8 \pi} P(\lambda) \sin ^{2} \theta \sin ^{2} \phi \quad I_{p}^{(\mathrm{p})}(\lambda)=\frac{3}{8 \pi} P(\lambda)(\cos \theta \sin \alpha-\sin \theta \cos \phi \cos \alpha)^{2}$.

Consider now a luminescent layer of thickness $H$, and thus an ensemble of incoherently radiating electric dipoles having equal dipole moment $\boldsymbol{p}$ but randomly oriented. To take this random orientation of the dipoles into account, we have to average $I_{p}^{(\mathrm{s}, \mathrm{p})}$ over the direction of $\boldsymbol{p}$, i.e., $\theta$ and $\phi$. The intensity obtained is named $I_{\langle p\rangle}^{(\mathrm{s}, \mathrm{p})}$ :

$I_{\langle p\rangle}^{(\mathrm{s}, \mathrm{p})}(\alpha, \lambda)=\frac{1}{4 \pi} \int_{0}^{\pi} \int_{0}^{2 \pi} I_{p}^{(\mathrm{s}, \mathrm{p})}(\lambda) \sin \theta \mathrm{d} \theta \mathrm{d} \phi$

$I_{\langle p\rangle}^{(\mathrm{s})}(\alpha, \lambda)=I_{\langle p\rangle}^{(\mathrm{p})}(\alpha, \lambda)=\frac{1}{8 \pi} P(\lambda)$.

The dipoles are located in the layer of thickness $H$; the total intensity emitted from this luminescent layer is thus obtained by integrating the product of $I_{\langle p\rangle}^{(\mathrm{s}, \mathrm{p})}$ with $L_{z}(h)$, the linear density of the radiating dipoles $\left(m^{-1}\right)$, over the layer depth $h$. As the layer is formed by a homogenous medium, $L_{z}(h)$ is constant with $h$ :

$I^{(\mathrm{s}, \mathrm{p})}(\alpha, \lambda)=\int_{0}^{H} I_{\langle\mathrm{p}\rangle}^{(\mathrm{s}, \mathrm{p})}(\alpha, \lambda) \cdot L_{z}(h) \mathrm{d} h$
$I^{(\mathrm{s})}(\alpha, \lambda)=I^{(\mathrm{p})}(\alpha, \lambda)=\frac{L_{z}}{8 \pi} \int_{0}^{H} P(\lambda, h) \mathrm{d} h$
$I(\alpha, \lambda)=I^{(\mathrm{s})}(\alpha, \lambda)+I^{(\mathrm{p})}(\alpha, \lambda)=\frac{L_{z}}{4 \pi} \int_{0}^{H} P(\lambda, h) \mathrm{d} h$.

Let us consider now an absorbing medium with an index $\tilde{n}$ that is thus complex. The following equations show the effect of propagation of an electromagnetic wave (wave vector $\tilde{k}$ ) on a length $r$ in this medium of complex refractive index $\tilde{n}$

- on the wave amplitude $E$ :

$$
\begin{aligned}
& E^{\prime}=E \cdot \exp (\mathrm{i} \tilde{\boldsymbol{k}} \cdot \boldsymbol{r})=E \cdot \exp \left(\mathrm{i} \frac{2 \pi}{\lambda} \tilde{n} r\right) \\
& E^{\prime}=E \cdot \underbrace{\exp \left(\mathrm{i} \frac{2 \pi}{\lambda} n r\right)}_{\text {phase shift }} \cdot \underbrace{\exp \left(-\frac{2 \pi}{\lambda} \kappa r\right)}_{\text {absorption }=\mathrm{e}^{-\tau / 2}}
\end{aligned}
$$

- on the wave intensity $I$ :

$$
\begin{aligned}
& I^{\prime} \propto\left\|E^{\prime}\right\|^{2} \\
& I^{\prime}=I \cdot \exp \left(-\frac{4 \pi}{\lambda} \kappa \cdot r\right)=I \cdot \exp (-\alpha \cdot r)=I \cdot \exp (-\tau)
\end{aligned}
$$

where $\alpha$ is the absorption coefficient of the medium $\left(\mathrm{in}^{-1}\right.$ ) and $\tau$ is the optical thickness. 


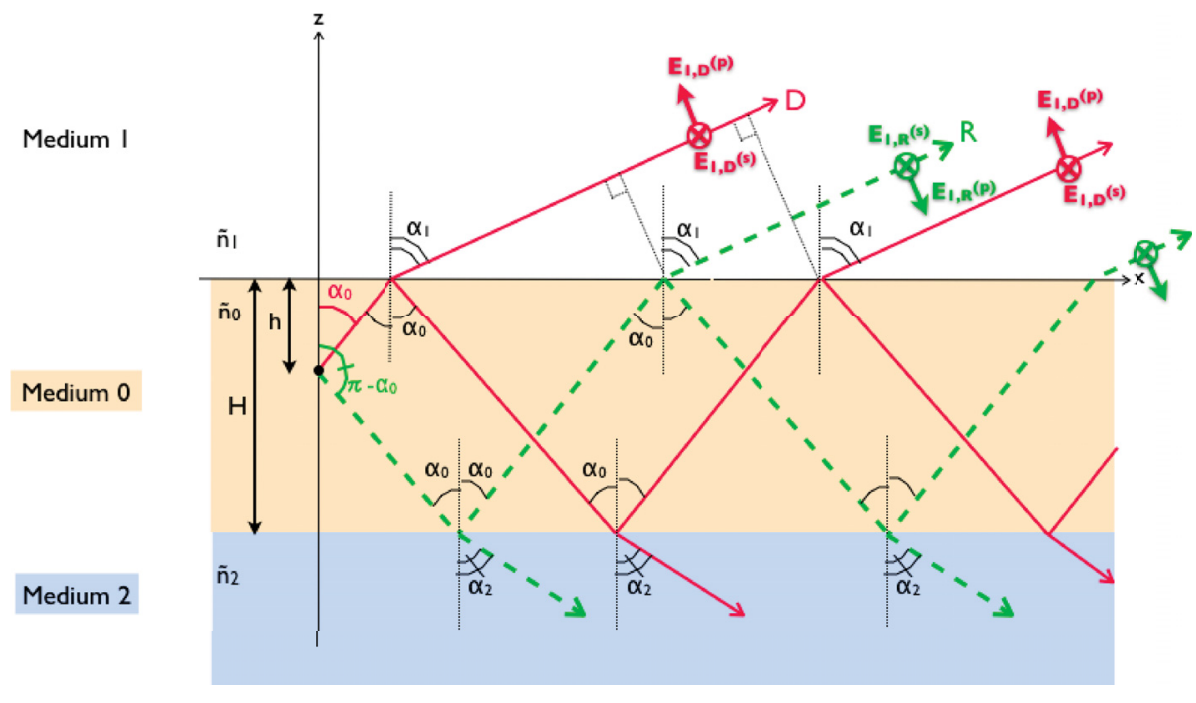

Fig. B.1. Schematic diagram of the emission geometry for a dipole embedded in a thin film (medium 0 ), at a distance $\mathrm{h}$ from the surface, for an emission angle $\alpha_{1}$ in medium 1 . This emission is the combinaison of light from the direct beam $\mathrm{D}$ (red solid line) and from the reflected beam $\mathrm{R}$ (green dashed line).

\section{Appendix B: Effects of the interfaces of the film}

In this appendix, we introduce the effects of the interfaces of the film and calculate the emission amplitude in a medium 1 by summing all the transmitted components (of each polarization), taking the phase shift and absorption due to propagation in each medium into account. As a result, the radiation pattern are obtained: it is the angular distribution of the light emitted by the film into the half-space 1.

Until now, we have considered an infinite medium. We now consider three different homogenous media (medium 0 is a film between semi-infinite media 1 and 2) and their interfaces. The emitting dipole $\boldsymbol{p}$ is now located in the film 0 . Our aim is to determine the radiation transmitted in medium 1 under the angle of observation $\alpha_{1}$.

We separate the light transmitted in medium 1 into two beams: the direct beam D is the one emitted directly in the direction of medium 1 (with an emission angle $\alpha_{0}$ given by the angle of observation $\alpha_{1}$ and the Snell-Descartes relation of refraction). The reflected beam $R$ is the beam that is first directed toward medium 2 and reflected off the interface $0 / 2$ before being transmitted in medium 1. Each of these beams is reflected multiple times between each interface of the film 0 . In this paragraph, we see that interference effects appear because of these multiple reflections and also because of the sum of the two different beams, $D$ and $R$. This latter case of interference is known as the wide-angle effect.

We now consider that the medium 1 is not absorbing, this means that

$$
\kappa_{1}=0 \quad \tilde{n}_{1}=n_{1}
$$

\section{B.1. The direct beam $D$}

We first consider the the direct beam $\mathrm{D}$, with its multiple reflection back and forth in layer 0 , transmitted in medium 1 as shown in Fig. B.2:

$$
\begin{aligned}
E_{D}^{(\mathrm{s}, \mathrm{p})}= & E^{(\mathrm{s}, \mathrm{p})}\left(\alpha=\alpha_{0}\right) \\
E_{1, D}^{(\mathrm{s}, \mathrm{p})}= & E_{D}^{(\mathrm{s}, \mathrm{p})} \cdot \exp \left(\mathrm{i} \tilde{k}_{0} \frac{h}{\cos \alpha_{0}}\right) \cdot t_{01}^{(\mathrm{s}, \mathrm{p})} \\
& \times\left[1+(r_{01}^{(\mathrm{s}, \mathrm{p})} r_{02}^{(\mathrm{s}, \mathrm{p})} \exp \left(\mathrm{i} 2 \tilde{k}_{0} \frac{H}{\cos \alpha_{0}}\right) \cdot \underbrace{\exp \left(-\mathrm{i} \tilde{k}_{1} 2 H \tan \alpha_{0} \sin \alpha_{1}\right)}_{\text {external phase shift }})+(\ldots)^{2}+\ldots\right] \\
E_{1, D}^{(\mathrm{s}, \mathrm{p})}= & E_{D}^{(\mathrm{s}, \mathrm{p})} \cdot t_{01}^{(\mathrm{s}, \mathrm{p})} \cdot \exp \left(\mathrm{i} \delta_{0}(h)\right) \cdot \exp \left(-\tau_{0}(h) / 2\right) \\
& \times\left[1+\sum_{l=1}^{\infty}\left(r_{01}^{(\mathrm{s}, \mathrm{p})} r_{02}^{(\mathrm{s}, \mathrm{p})} \exp \left(\mathrm{i} 2 \delta_{0}(H)\right) \exp \left(-\tau_{0}(H)\right) \exp \left(\mathrm{i} \delta_{1}(H)\right)\right)^{l}\right] .
\end{aligned}
$$

The following notations are used to lighten expressions. 
M. Godard and E. Dartois: Photoluminescence of hydrogenated amorphous carbons

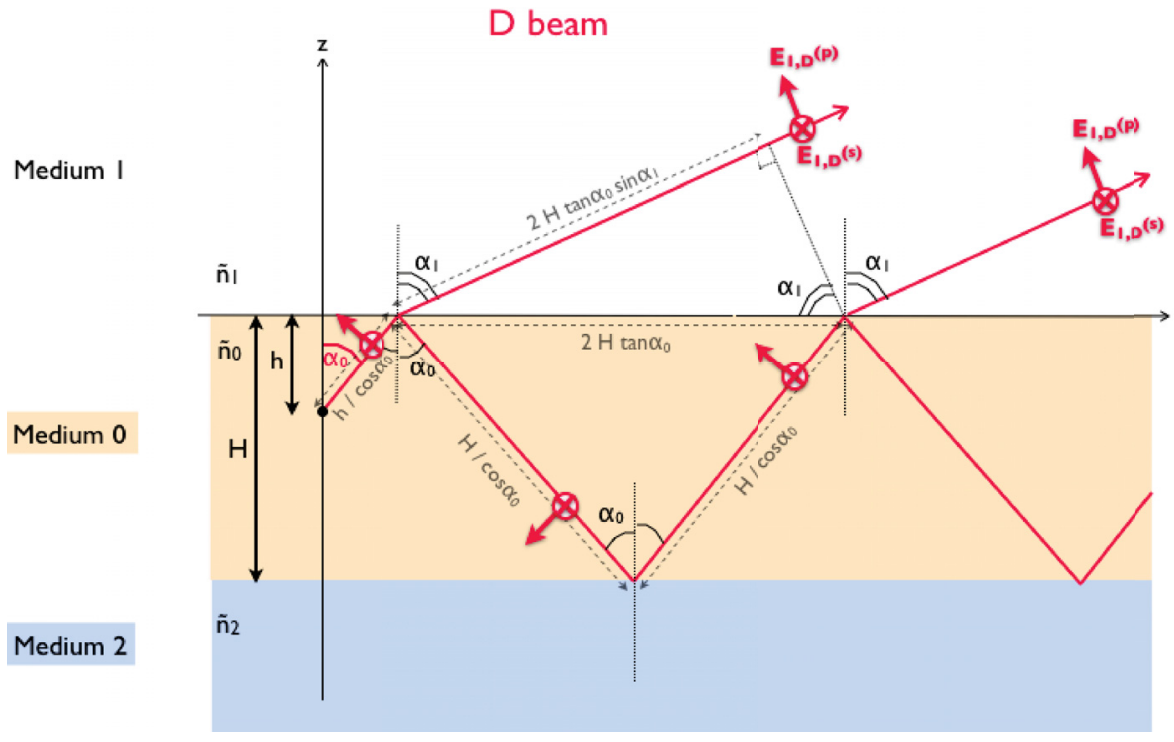

Fig. B.2. Schematic diagram of the emission geometry for the direct beam D.

Remark: $\tau_{0}$ is defined with a factor $\frac{1}{2}$ to obtain a power attenuation of $\mathrm{e}^{-\tau_{0}}$.

absorption:

$\frac{\tau_{0}(h)}{2}=\frac{2 \pi}{\lambda} \kappa_{0} \frac{h}{\cos \alpha_{0}}$

phase shift:

$\delta_{0}(h)=\frac{2 \pi}{\lambda} n_{0} \frac{h}{\cos \alpha_{0}}$

$\delta_{1}(h)=-\frac{2 \pi}{\lambda} n_{1} 2 h \tan \alpha_{0} \sin \alpha_{1}=-\frac{4 \pi}{\lambda} n_{0} h \frac{\sin ^{2} \alpha_{0}}{\cos \alpha_{0}}$

$\delta(h)=2 \delta_{0}(h)+\delta_{1}(h)=\frac{4 \pi}{\lambda} n_{0} h \cos \alpha_{0}$

$r_{i j}^{(\mathrm{s}, \mathrm{p})}=\rho_{i j}^{(\mathrm{s}, \mathrm{p})} \exp \left(\mathrm{i} \delta_{i j}^{(\mathrm{s}, \mathrm{p})}\right) \quad:$ Fresnel reflection coefficients

$\Delta^{(\mathrm{s}, \mathrm{p})}(h)=\delta(h)+\delta_{01}^{(\mathrm{s}, \mathrm{p})}+\delta_{02}^{(\mathrm{s}, \mathrm{p})}=2 \delta_{0}(h)+\delta_{1}(h)+\delta_{01}^{(\mathrm{s}, \mathrm{p})}+\delta_{02}^{(\mathrm{s}, \mathrm{p})}$,

where $\Delta(H)$ and $\tau(H)$ are the phase shift and the absorption due to only one wave back and forth in layer 0 before being transmitted in medium 1.

Hence, one derives

$E_{1, D}^{(\mathrm{s}, \mathrm{p})}=E_{D}^{(\mathrm{s}, \mathrm{p})} \cdot t_{01}^{(\mathrm{s}, \mathrm{p})} \cdot \exp \left(\mathrm{i} \delta_{0}(h)\right) \cdot \exp \left(-\tau_{0}(h) / 2\right) \cdot m^{(\mathrm{s}, \mathrm{p})}$

with: $\quad m^{(\mathrm{s}, \mathrm{p})}=\frac{1}{\left[1-\rho_{01}^{(\mathrm{s}, \mathrm{p})} \rho_{02}^{(\mathrm{s}, \mathrm{p})} \cdot \exp \left(\mathrm{i} \Delta^{(\mathrm{s}, \mathrm{p})}(H)\right) \cdot \exp \left(-\tau_{0}(H)\right)\right]}$.

\section{B.2. The reflected beam $R$}

For the reflected beam (see Fig. B.3), the factor due to the multiple reflections in the film stay the same as for the direct beam. We must keep in mind that the phase reference in medium 1 is defined by the direct beam:

$E_{R}^{(\mathrm{s}, \mathrm{p})}=E^{(\mathrm{s}, \mathrm{p})}\left(\alpha=\pi-\alpha_{0}\right)$

$$
\begin{aligned}
E_{1, R}^{(\mathrm{s}, \mathrm{p})}= & E_{R}^{(\mathrm{s,p})} \exp \left(\mathrm{i}_{\tilde{k}_{0}} \frac{H-h}{\cos \alpha_{0}}\right) r_{02}^{(\mathrm{s,p})} \exp \left(\mathrm{i} \tilde{k}_{0} \frac{H}{\cos \alpha_{0}}\right) t_{01}^{(\mathrm{s}, \mathrm{p})} \exp \left(-\mathrm{i} \tilde{k}_{1} 2(H-h) \tan \alpha_{0} \sin \alpha_{1}\right) \\
& \times\left[1+\left(r_{01}^{(\mathrm{s}, \mathrm{p})} r_{02}^{(\mathrm{s}, \mathrm{p})} \exp \left(\mathrm{i} 2 \tilde{k}_{0} \frac{H}{\cos \alpha_{0}}\right) \cdot \exp \left(-\mathrm{i} \tilde{k}_{1} 2 H \tan \alpha_{0} \sin \alpha_{1}\right)\right)+(\ldots)^{2}+\ldots\right] \\
E_{1, R}^{(\mathrm{s}, \mathrm{p})}= & E_{R}^{(\mathrm{s,p})} \cdot t_{01}^{(\mathrm{s,p})} r_{02}^{(\mathrm{s}, \mathrm{p})} \cdot \exp \left[\mathrm{i}\left(\delta_{0}(H-h)+\delta_{0}(H)+\delta_{1}(H-h)\right)\right] \\
& \times \exp \left[-\frac{1}{2}\left(\tau_{0}(H-h)+\tau_{0}(H)\right)\right] \cdot m^{(\mathrm{s}, \mathrm{p})} \\
E_{1, R}^{(\mathrm{s}, \mathrm{p})}= & E_{R}^{(\mathrm{s,p})} \cdot t_{01}^{(\mathrm{s,p})} r_{02}^{(\mathrm{s}, \mathrm{p})} \cdot \mathrm{e}^{\mathrm{i}\left(\delta_{0}(2 H-h)+\delta_{1}(H-h)\right)} \cdot \mathrm{e}^{-\frac{1}{2}\left(\tau_{0}(2 H-h)\right)} \cdot m^{(\mathrm{s}, \mathrm{p})} .
\end{aligned}
$$




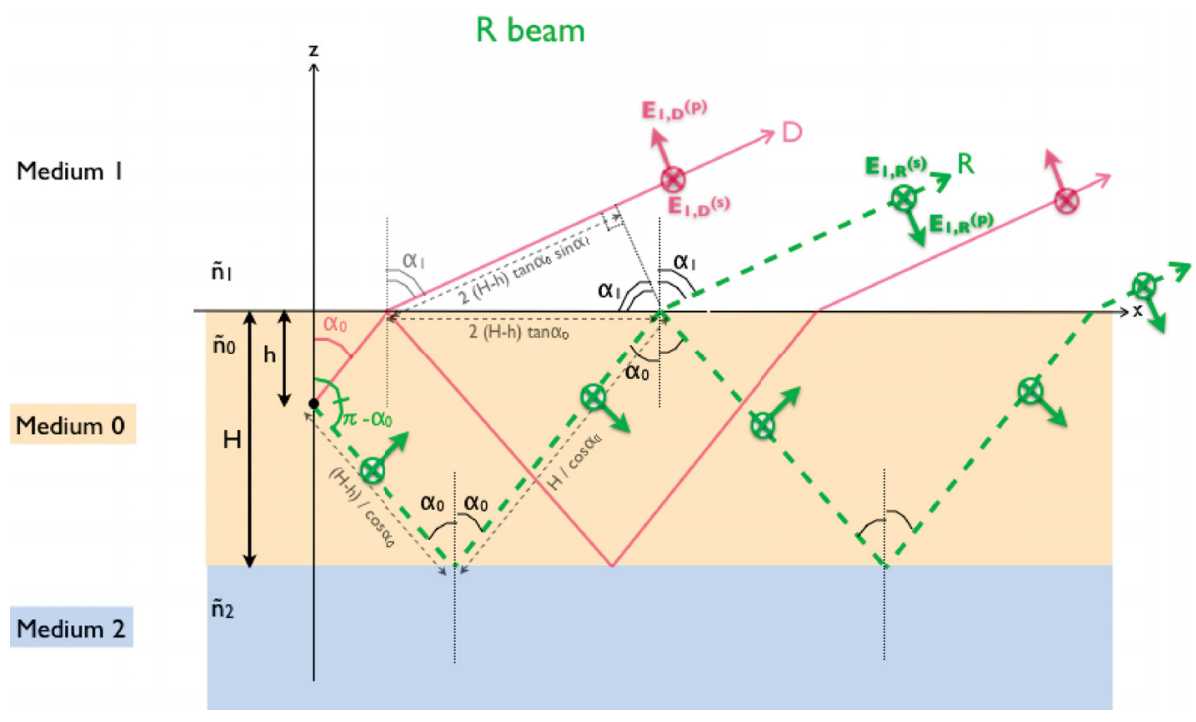

Fig. B.3. Schematic diagram of the emission geometry of the reflected beam R.

\section{B.3. Combination of the $D$ and $R$ beams}

We calculated the expression of the electric field amplitude transmitted in medium 1 of the direct beam $E_{1, D}^{(\mathrm{s}, \mathrm{p})}$ and of the reflected beam $E_{1, R}^{(\mathrm{s}, \mathrm{p})}$, for each polarization, (s) and (p). These amplitudes are exprimed as a function of $E_{D}^{(\mathrm{s}, \mathrm{p})}$ and $E_{R}^{(\mathrm{s}, \mathrm{p})}$, which are the electric field amplitude emitted by an electric dipole in the direction $\alpha_{D}=\alpha_{0}$ and $\alpha_{R}=\pi-\alpha_{0}$, respectively:

$E_{D}^{(\mathrm{s}, \mathrm{p})}=E^{(\mathrm{s}, \mathrm{p})}\left(\alpha=\alpha_{0}\right) \quad$ and: $\quad E_{R}^{(\mathrm{s}, \mathrm{p})}=E^{(\mathrm{s}, \mathrm{p})}\left(\alpha=\pi-\alpha_{0}\right)$.

The Eqs. (A.9) and (A.10) give the influence of the angle $\alpha$ on $E^{(\mathrm{s}, \mathrm{p})}$. One obtain

$E_{D}^{(\mathrm{s})}=E_{R}^{(\mathrm{s})}=E^{(\mathrm{s})}=\frac{E}{\sin \beta} \sin \theta \sin \phi$

$E_{D}^{(\mathrm{p})}=\frac{E}{\sin \beta}\left(\cos \theta \sin \alpha_{0}-\sin \theta \cos \phi \cos \alpha_{0}\right)$

$E_{R}^{(\mathrm{p})}=\frac{E}{\sin \beta}\left(\cos \theta \sin \left(\pi-\alpha_{0}\right)-\sin \theta \cos \phi \cos \left(\pi-\alpha_{0}\right)\right)$

$E_{R}^{(\mathrm{p})}=\frac{E}{\sin \beta}\left(\cos \theta \sin \alpha_{0}+\sin \theta \cos \phi \cos \alpha_{0}\right)$

$\Rightarrow E_{R}^{(\mathrm{p})} \neq E_{D}^{(\mathrm{p})}$.

To combine the electric fields in medium 1 due to the direct and reflected beams $\boldsymbol{E}_{1, D}^{(\mathrm{s}, \mathrm{p})}$ and $\boldsymbol{E}_{1, R}^{(\mathrm{s}, \mathrm{p})}$, we have to pay attention to the direction of these vectors and not only to their amplitude,

$\boldsymbol{E}_{1}^{(\mathrm{s}, \mathrm{p})}=\boldsymbol{E}_{1, D}^{(\mathrm{s}, \mathrm{p})}+\boldsymbol{E}_{1, R}^{(\mathrm{s}, \mathrm{p})}$

Let us consider the direction of the vectors $\boldsymbol{E}_{D}^{(\mathrm{s})}, \boldsymbol{E}_{D}^{(\mathrm{p})}, \boldsymbol{E}_{R}^{(\mathrm{s})}$ and $\boldsymbol{E}_{R}^{(\mathrm{p})}$ in medium 0, before any reflection or transmission at the film interfaces. The Eqs. (A.3) and (A.4) show that

$\boldsymbol{E}_{D}^{(\mathrm{s})}=\boldsymbol{E}_{R}^{(\mathrm{s})} \quad$ and $\quad \mid \begin{aligned} & E_{D, x}^{(\mathrm{p})}=-E_{R, x}^{(\mathrm{p})} \\ & E_{D, z}^{(\mathrm{p})}=E_{R, z^{\prime}}^{(\mathrm{p})}\end{aligned}$

The reflection at the interface 0/2 of the reflected beam changes the sign of $E_{R, z}^{(\mathrm{p})}$, so the direction of $\boldsymbol{E}_{D}^{(\mathrm{p})}$ and $\boldsymbol{E}_{R}^{(\mathrm{p})}$ become opposite. As one can see in Figs. B.1-B.3, the direct and reflected beam transmitted in medium 1, $\boldsymbol{E}_{1, D}$ and $\boldsymbol{E}_{1, R}$, respectively, are

- in the same direction for the s-polarization

- in opposite direction for the p-polarization. 
M. Godard and E. Dartois: Photoluminescence of hydrogenated amorphous carbons

As a result, the combination of the direct and reflected beams amplitude is done as

$E_{1}^{(\mathrm{s})}=E_{1, D}^{(\mathrm{s})}+E_{1, R}^{(\mathrm{s})} \quad$ and: $\quad E_{1}^{(\mathrm{p})}=E_{1, D}^{(\mathrm{p})}-E_{1, R}^{(\mathrm{p})}$

$E_{1}^{(\mathrm{s}, \mathrm{p})}=t_{01}^{(\mathrm{s}, \mathrm{p})} \cdot m^{(\mathrm{s}, \mathrm{p})}\left[E_{D}^{(\mathrm{s}, \mathrm{p})} \cdot \exp \left(\mathrm{i} \delta_{0}(h)\right) \cdot \exp \left(-\tau_{0}(h) / 2\right)\right.$

$\left.\pm_{(p)}^{(\mathrm{s})} E_{R}^{(\mathrm{s}, \mathrm{p})} \cdot r_{02}^{(\mathrm{s}, \mathrm{p})} \cdot \exp \left[\mathrm{i}\left(\delta_{0}(2 H-h)+\delta_{1}(H-h)\right)\right] \cdot \exp \left[-\frac{1}{2} \tau_{0}(2 H-h)\right]\right]$

$E_{1}^{(\mathrm{s}, \mathrm{p})}=t_{01}^{(\mathrm{s}, \mathrm{p})} m^{(\mathrm{s}, \mathrm{p})} \exp \left(\mathrm{i} \delta_{0}(h)\right) \exp \left(-\frac{\tau_{0}(h)}{2}\right)\left[E_{D}^{(\mathrm{s}, \mathrm{p})} \pm E_{R}^{(\mathrm{s}, \mathrm{p})} \rho_{02}^{(\mathrm{s}, \mathrm{p})} \exp \left(\mathrm{i} \delta_{w}^{(\mathrm{s}, \mathrm{p})}\right) \exp \left(-\frac{\tau_{w}}{2}\right)\right]$

where

$$
\delta_{w}^{(\mathrm{s}, \mathrm{p})}=2 \delta_{0}(H-h)+\delta_{1}(H-h)+\delta_{02}^{(\mathrm{s}, \mathrm{p})} \quad \text { and } \quad \tau_{w}=2 \tau_{0}(H-h) .
$$

\section{B.3.1. s-polarized}

$E_{1}^{(\mathrm{s})}=E^{(\mathrm{s})} \cdot \exp \left(\mathrm{i} \delta_{0}(h)\right) \cdot \exp \left(-\frac{\tau_{0}(h)}{2}\right) \cdot t_{01}^{(\mathrm{s})} \cdot m^{(\mathrm{s})} \cdot w_{+}^{(\mathrm{s})}$

$E_{1}^{(\mathrm{s})}=\frac{E}{\sin \beta} \sin \theta \sin \phi \cdot \exp \left(\mathrm{i} \delta_{0}(h)\right) \cdot \exp \left(-\frac{\tau_{0}(h)}{2}\right) \cdot t_{01}^{(\mathrm{s})} \cdot m^{(\mathrm{s})} \cdot w_{+}^{(\mathrm{s})}$

where: $\quad w_{+}^{(\mathrm{s})}=\left[1+\rho_{02}^{(\mathrm{s})} \cdot \exp \left(\mathrm{i} \delta_{\mathrm{w}}^{(\mathrm{s})}\right) \cdot \exp \left(-\frac{\tau_{\mathrm{w}}}{2}\right)\right]$

\section{B.3.2. p-polarized}

$E_{1}^{(\mathrm{p})}=\exp \left(\mathrm{i} \delta_{0}(h)\right) \exp \left(-\frac{\tau_{0}(h)}{2}\right) t_{01}^{(\mathrm{p})} m^{(\mathrm{p})}\left[E_{D}^{(\mathrm{p})}-E_{R}^{(\mathrm{p})} \cdot \rho_{02}^{(\mathrm{p})} \cdot \exp \left(\mathrm{i} \delta_{\mathrm{w}}^{(\mathrm{p})}\right) \cdot \exp \left(-\frac{\tau_{\mathrm{w}}}{2}\right)\right]$

$E_{1}^{(\mathrm{p})}=\frac{E}{\sin \beta} \mathrm{e}^{\mathrm{i} \delta_{0}(h)} \mathrm{e}^{-\frac{\tau_{0}(h)}{2}} t_{01}^{(\mathrm{p})} m^{(\mathrm{p})}\left[\cos \theta \sin \alpha_{0} w_{-}^{(\mathrm{p})}-\sin \theta \cos \phi \cos \alpha_{0} w_{+}^{(\mathrm{p})}\right]$

$w_{+}^{(\mathrm{p})}=\left[1+\rho_{02}^{(\mathrm{p})} \cdot \mathrm{e}^{\mathrm{i} \delta_{w}^{(\mathrm{p})}} \cdot \mathrm{e}^{-\frac{\tau_{W}}{2}}\right] \quad$ and $\quad w_{-}^{(\mathrm{p})}=\left[1-\rho_{02}^{(\mathrm{p})} \cdot \mathrm{e}^{\mathrm{i} \delta_{w}^{(\mathrm{p})}} \cdot \mathrm{e}^{-\frac{\tau_{W}}{2}}\right]$.

\section{B.4. Radiation pattern}

The radiation pattern is the angular distribution of the light emitted by the source $\boldsymbol{p}$ located in layer 0 into the half-space 1 , expressed by $I_{1, h, p(\theta, \phi)}^{(\mathrm{s}, \mathrm{p})}\left(\alpha_{0}, \phi, \lambda\right)$ the intensity $\left(W \cdot s r^{-1} \cdot n m^{-1}\right)$ emitted in the direction given the angles $\left(\alpha_{0}, \phi\right)$ into the half-space 1 .

$I_{1, h, p(\theta, \phi)}^{(\mathrm{s})}\left(\alpha_{0}, \phi, \lambda\right)=\frac{3}{8 \pi} P(\lambda, h) \sin ^{2} \theta \sin ^{2} \phi \cdot\left\|\exp \left(\mathrm{i} \delta_{0}(h)\right) \cdot \exp \left(-\frac{\tau_{0}(h)}{2}\right) \cdot t_{01}^{(\mathrm{s})} \cdot m^{(\mathrm{s})} \cdot w_{+}^{(\mathrm{s})}\right\|^{2} \cdot \frac{\mathrm{d} \Omega_{0}}{\mathrm{~d} \Omega_{1}}$

$I_{1, h, \boldsymbol{p}(\theta, \phi)}^{(\mathrm{s})}\left(\alpha_{0}, \phi, \lambda\right)=\frac{3}{8 \pi} P(\lambda, h) \sin ^{2} \theta \sin ^{2} \phi \cdot \mathrm{e}^{-\tau_{0}(h)} \cdot T_{01}^{(\mathrm{s})} \cdot\left\|m^{(\mathrm{s})}\right\|^{2} \cdot\left\|w_{+}^{(\mathrm{s})}\right\|^{2} \cdot \frac{\mathrm{d} \Omega_{0}}{\mathrm{~d} \Omega_{1}}$

$I_{1, h, \boldsymbol{p}(\theta, \phi)}^{(\mathrm{p})}\left(\alpha_{0}, \phi, \lambda\right)=\frac{3}{8 \pi} P(\lambda, h) \mathrm{e}^{-\tau_{0}(h)} T_{01}^{(\mathrm{p})}\left\|m^{(\mathrm{p})}\right\|^{2} \frac{\mathrm{d} \Omega_{0}}{\mathrm{~d} \Omega_{1}}\left\|\cos \theta \sin \alpha_{0} w_{-}^{(\mathrm{p})}-\sin \theta \cos \phi \cos \alpha_{0} w_{+}^{(\mathrm{p})}\right\|^{2}$

$I_{1, h, \boldsymbol{p}(\theta, \phi)}^{(\mathrm{p})}\left(\alpha_{0}, \phi, \lambda\right)=\frac{3}{8 \pi} P(\lambda, h) \mathrm{e}^{-\tau_{0}(h)} T_{01}^{(\mathrm{p})}\left\|m^{(\mathrm{p})}\right\|^{2} \frac{\mathrm{d} \Omega_{0}}{\mathrm{~d} \Omega_{1}}$

$$
\times\left[\cos ^{2} \theta \sin ^{2} \alpha_{0}\left\|w_{-}^{(\mathrm{p})}\right\|^{2}+\sin ^{2} \theta \cos ^{2} \phi \cos ^{2} \alpha_{0}\left\|w_{+}^{(\mathrm{p})}\right\|^{2}-\frac{1}{2} \sin 2 \theta \cos \phi \sin 2 \alpha_{0} w_{0}^{(\mathrm{p})}\right]
$$

where: $w_{0}^{(\mathrm{p})}=\mathfrak{R e}\left(w_{-}^{(\mathrm{p})}\right) \mathfrak{R e}\left(w_{+}^{(\mathrm{p})}\right)+\mathfrak{I m}\left(w_{-}^{(\mathrm{p})}\right) \mathfrak{I m}\left(w_{+}^{(\mathrm{p})}\right)=1-\rho_{02}^{(p) 2} \cdot \exp \left(-\tau_{w}\right)$.

\section{B.4.1. Solid angle modification at the interface}

The ratio $\frac{\mathrm{d} \Omega_{0}}{\mathrm{~d} \Omega_{1}}$ represents the modification of solid angle when the medium changes, $I_{i} \mathrm{~d} \Omega_{i}$ is the radiated power in medium $i$ into the solid angle $\mathrm{d} \Omega_{i}$. When the wave goes through the interface $i / j$, the solid angle is modified and the power conservation is given by Eq. (B.12) (when the transmission coefficient is 1):

$I_{i} \mathrm{~d} \Omega_{i}=I_{j} \mathrm{~d} \Omega_{j}$. 
This is the origin of the factor $\frac{\mathrm{d} \Omega_{0}}{\mathrm{~d} \Omega_{1}}$ in the expression of the intensity $I_{1}^{(\mathrm{s}, \mathrm{p})}$,

$\frac{\mathrm{d} \Omega_{0}}{\mathrm{~d} \Omega_{1}}=\frac{\sin \alpha_{0} \mathrm{~d} \alpha_{0} \mathrm{~d} \phi}{\sin \alpha_{1} \mathrm{~d} \alpha_{1} \mathrm{~d} \phi}=\frac{n_{1}^{2} \cos \alpha_{1}}{n_{0}^{2} \cos \alpha_{0}}$

using the derivative of the Snell-Descartes law.

\section{B.4.2. Random orientation of the dipoles}

To take a random orientation for the electric dipoles embedded in the film account, the intensity is averaged over $\theta$ and $\phi$. The geometry is thus no more dependent on the angle $\phi ; I_{1}$ is now only depending on the angle of observation $\alpha_{0}$. $P(\lambda, h)$, which is the total power emitted in all directions of the space, at the wavelength $\lambda$, by a single dipole $\boldsymbol{p}$ at the depth $h$, does not vary with the direction of the dipole.

The intensity ( $W s r^{-1} m^{-1} m^{-1}$ ) emitted by a film layer of thickness $\mathrm{d} h$, at the film depth $h$, is

$$
\begin{aligned}
& I_{1, h}^{(\mathrm{s}, \mathrm{p})}\left(\alpha_{0}, \lambda\right)=\frac{1}{4 \pi} \int_{0}^{\pi} \int_{0}^{2 \pi} L_{z}(h) I_{1, h, p(\theta, \phi)}^{(\mathrm{s}, \mathrm{p})}\left(\alpha_{0}, \phi, \lambda\right) \sin \theta \mathrm{d} \theta \mathrm{d} \phi \\
& I_{1, h}^{(\mathrm{s})}\left(\alpha_{0}, \lambda\right)=\frac{3}{32 \pi^{2}} L_{z}(h) P(\lambda, h) \cdot \mathrm{e}^{-\tau_{0}(h)} \cdot T_{01}^{(\mathrm{s})} \cdot\left\|m^{(\mathrm{s})}\right\|^{2} \cdot\left\|w_{+}^{(\mathrm{s})}\right\|^{2} \cdot \frac{\mathrm{d} \Omega_{0}}{\mathrm{~d} \Omega_{1}} \cdot \int_{0}^{\pi} \sin ^{3} \theta \mathrm{d} \theta \int_{0}^{2 \pi} \sin ^{2} \phi \mathrm{d} \phi \\
& I_{1, h}^{(\mathrm{s})}\left(\alpha_{0}, \lambda\right)=\frac{3}{32 \pi^{2}} L_{z}(h) P(\lambda, h) \mathrm{e}^{-\tau_{0}(h)} T_{01}^{(\mathrm{s})}\left\|m^{(\mathrm{s})}\right\|^{2}\left\|w_{+}^{(\mathrm{s})}\right\|^{2} \frac{\mathrm{d} \Omega_{0}}{\mathrm{~d} \Omega_{1}} \cdot\left[-\cos \theta+\frac{\cos ^{3} \theta}{3}\right]_{0}^{\pi}\left[\frac{\phi}{2}-\frac{\sin (2 \phi)}{4}\right]_{0}^{2 \pi} \\
& I_{1, h}^{(\mathrm{s})}\left(\alpha_{0}, \lambda\right)=\frac{1}{8 \pi} L_{z}(h) P(\lambda, h) \mathrm{e}^{-\tau_{0}(h)} T_{01}^{(\mathrm{s})}\left\|m^{(\mathrm{s})}\right\|^{2}\left\|w_{+}^{(\mathrm{s})}\right\|^{2} \frac{\mathrm{d} \Omega_{0}}{\mathrm{~d} \Omega_{1}} \\
& I_{1, h}^{(\mathrm{p})}\left(\alpha_{0}, \lambda\right)=\frac{3}{32 \pi^{2}} L_{z}(h) P(\lambda, h) \mathrm{e}^{-\tau_{0}(h)} T_{01}^{(\mathrm{p})}\left\|m^{(\mathrm{p})}\right\|^{2} \frac{\mathrm{d} \Omega_{0}}{\mathrm{~d} \Omega_{1}} \cdot\left[\sin ^{2} \alpha_{0}\left\|w_{-}^{(\mathrm{p})}\right\|^{2} \int_{0}^{\pi} \cos ^{2} \theta \sin \theta \mathrm{d} \theta \int_{0}^{2 \pi} \mathrm{d} \phi\right. \\
& \left.+\cos ^{2} \alpha_{0}\left\|w_{+}^{(\mathrm{p})}\right\|^{2} \int_{0}^{\pi} \sin ^{3} \theta \mathrm{d} \theta \int_{0}^{2 \pi} \cos ^{2} \phi \mathrm{d} \phi-\frac{1}{2} \sin 2 \alpha_{0} w_{0}^{(\mathrm{p})} \int_{0}^{\pi} \sin 2 \theta \sin \theta \mathrm{d} \theta \int_{0}^{2 \pi} \cos \phi \mathrm{d} \phi\right] \\
& I_{1, h}^{(\mathrm{p})}\left(\alpha_{0}, \lambda\right)=\frac{3}{32 \pi^{2}} L_{z}(h) P(\lambda, h) \mathrm{e}^{-\tau_{0}(h)} T_{01}^{(\mathrm{p})}\left\|m^{(\mathrm{p})}\right\|^{2} \frac{\mathrm{d} \Omega_{0}}{\mathrm{~d} \Omega_{1}} \cdot\left[\sin ^{2} \alpha_{0}\left\|w_{-}^{(\mathrm{p})}\right\|^{2} \frac{2}{3} 2 \pi+\cos ^{2} \alpha_{0}\left\|w_{+}^{(\mathrm{p})}\right\|^{2} \frac{4}{3} \pi\right] \\
& I_{1, h}^{(\mathrm{p})}\left(\alpha_{0}, \lambda\right)=\frac{1}{8 \pi} L_{z}(h) P(\lambda, h) \mathrm{e}^{-\tau_{0}(h)} T_{01}^{(\mathrm{p})}\left\|m^{(\mathrm{p})}\right\|^{2} \frac{\mathrm{d} \Omega_{0}}{\mathrm{~d} \Omega_{1}} \cdot\left[\sin ^{2} \alpha_{0}\left\|w_{-}^{(\mathrm{p})}\right\|^{2}+\cos ^{2} \alpha_{0}\left\|w_{+}^{(\mathrm{p})}\right\|^{2}\right] .
\end{aligned}
$$

Finally, the specific radiant intensity in medium 1 and created by electric dipoles of random orientation at the film depth $h$ is

$$
I_{1, h}^{(\mathrm{s}, \mathrm{p})}\left(\alpha_{0}, \lambda\right)=\frac{1}{8 \pi} \mathrm{e}^{-\tau_{0}(h)} T_{01}^{(\mathrm{s}, \mathrm{p})} M^{(\mathrm{s}, \mathrm{p})} W^{(\mathrm{s}, \mathrm{p})} \frac{n_{1}^{2} \cos \alpha_{1}}{n_{0}^{2} \cos \alpha_{0}} L_{z}(h) P(\lambda, h)
$$

where $\quad M^{(\mathrm{s}, \mathrm{p})}=\left\|m^{(\mathrm{s}, \mathrm{p})}\right\|^{2}=\left\|1-\rho_{01}^{(\mathrm{s}, \mathrm{p})} \rho_{02}^{(\mathrm{s}, \mathrm{p})} \cdot \exp \left(\mathrm{i} \Delta^{(\mathrm{s}, \mathrm{p})}(H)\right) \cdot \exp \left(-\tau_{0}(H)\right)\right\|^{-2}$

$$
=1+\rho_{01}^{(\mathrm{s}, \mathrm{p}) 2} \rho_{02}^{(\mathrm{s}, \mathrm{p}) 2} \cdot \mathrm{e}^{-2 \tau_{0}(H)}-2 \rho_{01}^{(\mathrm{s}, \mathrm{p})} \rho_{02}^{(\mathrm{s}, \mathrm{p})} \cdot \mathrm{e}^{-\tau_{0}(H)} \cos \left(\Delta^{(\mathrm{s}, \mathrm{p})}(H)\right)
$$

and $\quad W^{(\mathrm{s})}=\left\|w_{+}^{(\mathrm{s})}\right\|^{2}=\left\|1+\rho_{02}^{(\mathrm{s}, \mathrm{p})} \cdot \exp \left(\mathrm{i} \delta_{w}^{(\mathrm{s})}\right) \cdot \exp \left(-\frac{\tau_{w}}{2}\right)\right\|^{2}$

$$
=1+\rho_{02}^{(\mathrm{s}, \mathrm{p}) 2} \cdot \mathrm{e}^{-\tau_{w}}+2 \rho_{02}^{(\mathrm{s}, \mathrm{p})} \cdot \mathrm{e}^{-\tau_{w} / 2} \cos \left(\delta_{w}^{(\mathrm{s})}\right)
$$

$W^{(\mathrm{p})}=\sin ^{2} \alpha_{0}\left\|w_{-}^{(\mathrm{p})}\right\|^{2}+\cos ^{2} \alpha_{0}\left\|w_{+}^{(\mathrm{p})}\right\|^{2}$

$$
=1+\rho_{02}^{(\mathrm{s}, \mathrm{p}) 2} \mathrm{e}^{-\tau_{w}}+2 \rho_{02}^{(\mathrm{s}, \mathrm{p})} \mathrm{e}^{-\tau_{w} / 2} \cos \left(\delta_{w}^{(\mathrm{p})}\right) \cos \left(2 \alpha_{0}\right)
$$

recalling that $\quad \Delta^{(\mathrm{s}, \mathrm{p})}(h)=2 \delta_{0}(h)+\delta_{1}(h)+\delta_{01}^{(\mathrm{s}, \mathrm{p})}+\delta_{02}^{(\mathrm{s}, \mathrm{p})}=\frac{4 \pi}{\lambda} n_{0} h \cos \alpha_{0}+\delta_{01}^{(\mathrm{s}, \mathrm{p})}+\delta_{02}^{(\mathrm{s}, \mathrm{p})}$

$\delta_{w}^{(\mathrm{s}, \mathrm{p})}=2 \delta_{0}(H-h)+\delta_{1}(H-h)+\delta_{02}^{(\mathrm{s}, \mathrm{p})}=\frac{4 \pi}{\lambda} n_{0}(H-h) \cos \alpha_{0}+\delta_{02}^{(\mathrm{s}, \mathrm{p})}$

$\tau_{w}=2 \tau_{0}(H-h)=\frac{8 \pi}{\lambda} \kappa_{0} \frac{H-h}{\cos \alpha_{0}}$,

where $\left\|t_{01}^{(\mathrm{s}, \mathrm{p})}\right\|^{2}, \rho_{i j}^{(\mathrm{s}, \mathrm{p})}$ and $\delta_{i j}^{(\mathrm{s}, \mathrm{p})}$ are expressed in the next section. The variable $M^{(\mathrm{s}, \mathrm{p})}$ gives the influence of the multiple-beam interference, and $W^{(\mathrm{s}, \mathrm{p})}$ is due to the wide angle interference. 


\section{B.4.3. Radiant intensity observed in medium 1}

Until now, we have not being interested in the wavelength dependence of the emission. The observed intensity depends on $\lambda$ because each dipole radiates with a power $P(\lambda, h)$ that depends on the wavelength through an emission profile $\operatorname{Em}(\lambda)\left(\right.$ in $\left.\mathrm{nm}^{-1}\right)$ proper to the material of the film. In the case of photoluminescence, the emission of each dipole will also result in an excitation absorbed by the film $\left(\operatorname{Abs}(h)\right.$ is the power absorbed in unit of length $\left.\left(\mathrm{W} \cdot \mathrm{m}^{-1}\right)\right)$ that can depend on the film depth $h$. The emission also results in the photoluminescence yield $\eta_{\mathrm{E}}{ }^{8}$.

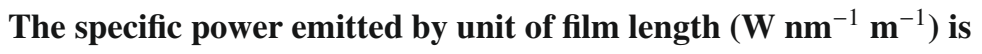

$L_{z}(h) P(\lambda, h)=\operatorname{Abs}(h) \eta_{\mathrm{E}} \operatorname{Em}(\lambda)$.

The total power $(W)$ between the wavelengths $\lambda_{\min }$ and $\lambda_{\max }$, emitted by the film in all directions in space is

$P_{\text {tot }}=\int_{\lambda_{\min }}^{\lambda_{\max }} \int_{0}^{H} L_{z}(h) P(\lambda, h) \mathrm{d} h \mathrm{~d} \lambda$

$P_{\text {tot }}=\eta_{\mathrm{E}} \int_{0}^{H} \operatorname{Abs}(h) \mathrm{d} h \int_{\lambda_{\min }}^{\lambda_{\max }} \operatorname{Em}(\lambda) \mathrm{d} \lambda$.

Moreover, because the whole film is radiating, the electric dipoles $p$ are located at all the film depths $h$ between 0 and $H$, with a constant distribution on this interval. Thus, to obtain the specific radiant intensity that is observed in medium 1 , we must integrate the expression given by the Eq. (B.15) on the interval $[0, H]$ :

$I_{1}^{(\mathrm{s}, \mathrm{p})}\left(\alpha_{0}, \lambda\right)=\int_{0}^{H} I_{1, h}^{(\mathrm{s}, \mathrm{p})}\left(\alpha_{0}, \lambda\right) \mathrm{d} h$

$I_{1}\left(\alpha_{0}, \lambda\right)=I_{1}^{(\mathrm{s})}\left(\alpha_{0}, \lambda\right)+I_{1}^{(\mathrm{p})}\left(\alpha_{0}, \lambda\right)$.

The total power between the wavelengths $\lambda_{\min }$ and $\lambda_{\max }$, received on a detector $D$ that "sees" an solid angle $\Omega_{D}$ is

$P_{1, D}\left(\alpha_{0}\right)=\int_{\Omega_{D}} \int_{\lambda_{\min }}^{\lambda_{\max }} I_{1}\left(\alpha_{0}, \lambda\right) \mathrm{d} \lambda \mathrm{d} \Omega$.

\section{Appendix C: Fresnel coefficients}

In this appendix, we express the Fresnel coefficients for an absorbing medium (meaning with a complex refractive index).

\section{C.1. Reflection}

The Fresnel reflection coefficients are given by the equations:

$r_{i j}^{(\mathrm{s})}=\frac{\tilde{n}_{i} \cos \alpha_{i}-\tilde{n}_{j} \cos \alpha_{j}}{\tilde{n}_{i} \cos \alpha_{i}+\tilde{n}_{j} \cos \alpha_{j}} \quad r_{i j}^{(\mathrm{p})}=\frac{\tilde{n}_{i} \cos \alpha_{j}-\tilde{n}_{j} \cos \alpha_{i}}{\tilde{n}_{i} \cos \alpha_{j}+\tilde{n}_{j} \cos \alpha_{i}}$

The angles $\alpha_{i, j}$ are here complex angles given by the Descartes-Snell law of refraction for absorbing media, i.e., with complex refractive indexes (Kovalenko 2001):

$\tilde{n}_{i} \sin \alpha_{i}=\tilde{n}_{j} \sin \alpha_{j}$

The variables $\rho_{i j}^{(\mathrm{s}, \mathrm{p})}$ and $\delta_{i j}^{(\mathrm{s}, \mathrm{p})}$ are defined by $r_{i j}^{(\mathrm{s}, \mathrm{p})}=\rho_{i j}^{(\mathrm{s}, \mathrm{p})} \cdot \mathrm{e}^{\mathrm{i} \delta_{i j}^{(\mathrm{s}, \mathrm{p})}}$.

\section{C.2. Transmission}

The Fresnel transmission coefficients (ratio of wave amplitudes) are given by the equations:

$t_{i j}^{(\mathrm{s}, \mathrm{p})}=\frac{E_{j}^{(\mathrm{s}, \mathrm{p})}}{E_{i}^{(\mathrm{s}, \mathrm{p})}}$

$t_{i j}^{(\mathrm{s})}=\frac{2 \tilde{n}_{i} \cos \alpha_{i}}{\tilde{n}_{i} \cos \alpha_{i}+\tilde{n}_{j} \cos \alpha_{j}} \quad t_{i j}^{(\mathrm{p})}=\frac{2 \tilde{n}_{i} \cos \alpha_{i}}{\tilde{n}_{i} \cos \alpha_{j}+\tilde{n}_{j} \cos \alpha_{i}}$.

8 The photoluminescence yield is here the energy (or power) ratio, not the quantum yield that is a photon number ratio. 
The Fresnel transmittances (ratio of powers) are given by the equations

$T_{i j}^{(\mathrm{s}, \mathrm{p})}=\frac{P_{j}^{(\mathrm{s}, \mathrm{p})}}{P_{i}^{(\mathrm{s}, \mathrm{p})}}=\frac{\operatorname{Irr}_{j}^{(\mathrm{s}, \mathrm{p})} A_{j}}{\operatorname{Irr}_{i}^{(\mathrm{s}, \mathrm{p})} A_{i}}$,

where $A_{i, j}$ is the surface section of the light beam in medium i or $\mathrm{j}$, and $P_{i, j}$ and $\operatorname{Irr}_{i, j}$ are the incident and transmitted power (in Watt) and the irradiance (in $\mathrm{W} \mathrm{m}^{-2}$ ):

$T_{i j}^{(\mathrm{s}, \mathrm{p})}=\frac{\operatorname{Irr}_{j}^{(\mathrm{s}, \mathrm{p})} \cos \alpha_{j}}{\operatorname{Irr}_{i}^{(\mathrm{s}, \mathrm{p})} \cos \alpha_{i}}$

$T_{i j}^{(\mathrm{s}, \mathrm{p})}=\frac{n_{j} \cos \alpha_{j}}{n_{i} \cos \alpha_{i}}\left\|t_{i j}^{(\mathrm{s}, \mathrm{p})}\right\|^{2}$.

Remark: In the same way, the reflectance is also defined as $\quad R_{i j}^{(\mathrm{s}, \mathrm{p})}=\frac{P_{i, \mathrm{reffected}}^{(\mathrm{s}, \mathrm{p})}}{P_{i}^{\mathrm{s}, \mathrm{p}}}=\left\|r_{i j}^{(\mathrm{s}, \mathrm{p})}\right\|^{2}$

$R_{i j}^{(\mathrm{s}, \mathrm{p})}+T_{i j}^{(\mathrm{s}, \mathrm{p})}=1$

We can also define another transmission factor $\hat{T}_{i j}$ that takes the modification of the solid angle due to refraction at the interface between media $\mathrm{i}$ and $\mathrm{j}$ into account:

$\hat{T}_{i j}^{(\mathrm{s}, \mathrm{p})}=T_{i j}^{(\mathrm{s}, \mathrm{p})} \frac{\mathrm{d} \Omega_{i}}{\mathrm{~d} \Omega_{j}}$

$\hat{T}_{i j}^{(\mathrm{s}, \mathrm{p})}=T_{i j}^{(\mathrm{s}, \mathrm{p})} \frac{n_{j}^{2} \cos \alpha_{j}}{n_{i}^{2} \cos \alpha_{i}}$

$\hat{T}_{i j}^{(\mathrm{s}, \mathrm{p})}=\left\|t_{i j}^{(\mathrm{s}, \mathrm{p})}\right\|^{2} \frac{n_{j}^{3} \cos ^{2} \alpha_{j}}{n_{i}^{3} \cos ^{2} \alpha_{i}}$. 